\title{
4 Digitale Medien und digitales Lernen
}

Dieses Kapitel wurde bei Erstveröffentlichung ohne die korrekte Creative Commons Lizenz veröffentlicht. Die korrekte Lizenz finden Sie am Ende des Kapitels.

Ein Erratum zu diesem Kapitel ist verfügbar unter DOI 10.1007/978-3-658-12533-2_14

(C) SINUS Markt- und Sozialforschung GmbH 2016

M. Calmbach et al., Wie ticken Jugendliche 2016?, DOI 10.1007/978-3-658-12533-2_4 
Digitalisierung ist ein wesentlicher Treiber des aktuellen Wertewandels und sozialer Transformationsprozesse. Gerade aufgrund des hohen Entwicklungstempos technischer Neuerungen und der Konsequenzen für zentrale gesellschaftliche Bereiche (Arbeitswelt, Bildung, Erziehung, Kommunikation, Alltagsorganisation, Mobilität etc.) sind die Auswirkungen umfassend und betreffen fast alle Facetten unserer Lebenswelt. Auch im Alltag von Jugendlichen sind digitale Medien heute nicht mehr wegzudenken. Sie nutzen nicht nur diverse Angebote und Services - sie leben online.

Inwieweit aber hat die Digitalisierung soziale Implikationen für das Heranwachsen von Jugendlichen? Um dieser Frage nachgehen zu können, ist ein breites Verständnis des digitalen Alltags von Jugendlichen erforderlich. Es reicht nicht, Aktivitätsmuster zu erfassen (wer macht was wann, wie oft und wo); von entscheidender Bedeutung ist vielmehr, wie Jugendliche selbst ihr digitales Leben beschreiben und bewerten und vor allem: warum ihnen bestimmte Medien bzw. Geräte so wichtig sind und was genau ihren Wert ausmacht.

In diesem Zusammenhang wird auch die Frage immer relevanter, ob und wie digitale Medien in der schulischen wie auch außerschulischen Bildung eingesetzt werden bzw. werden sollten. Dabei geht es aber nicht nur um das Lernen mit digitalen Medien, sondern auch das Lernen über digitale Medien, d. h. um den Erwerb zentraler Kompetenzen für einen selbstbestimmten, aktiv gestaltenden Umgang mit ihnen, jenseits reiner Rezeption von Online-Angeboten und Services. Ein weiterer Schwerpunkt dieses Kapitels wird daher auf den Bereich Medienkompetenz und "Digitales Lernen" gelegt. Auch der Blick auf mögliche Veränderungen von Bildungszielen und -chancen ist hierbei von enormer Bedeutung, denn dies entscheidet wesentlich darüber, wie nachfolgende Generationen Wissen erwerben, verarbeiten und weitergeben. Lernen - ob zu Hause oder in der Schule - findet immer selbstverständlicher mit bzw. via digitale Medien statt. Haben aber alle gleiche Möglicheiten, im digitalen Alltag zu bestehen? In den einschlägigen Medien wird gerade Chancengerechtigkeit als besonderes Potenzial hervorgehoben: Das Internet verspräche Mitbestimmung, Transparenz und Kooperation. Aber ist digitale Chancengerechtigkeit bereits Realität in jugendlichen Lebenswelten? Oder schreibt die Digitalisierung bestehende soziale Ungleichheiten fort? Diesen Fragen wird das folgende 
Kapitel nachgehen und darlegen, wie sich die persönliche Medienkompetenz aus Perspektive der Jugendlichen zusammensetzt und auf welche Art und Weise digitale Medien v. a. beim Lernen eingesetzt werden.

\subsection{Ausstattung mit und Zugänge zu digitalen Medien}

\section{Jugendliche sind mit Blick auf die digitale Ausstattung fast wunschlos glücklich.}

Dass Jugendliche medial umfassend ausgestattet sind, ist keine neue Erkenntnis - neu ist aber, dass Jugendliche das mittlerweile auch so sehen. Entscheidend ist hierbei weniger die absolute Medienausstattung, sondern die Vergleichsperspektive: Wer ein Smartphone, aber keine Spielekonsole hat, grenzt sich von denen ab, die "noch nicht mal" ein Smartphone haben; wer kein Smartphone hat, grenzt sich von denen $a b$, die noch nicht mal ein Handy haben.

Seit Jahrzehnten stehen elektronische Geräte auf den Wunschlisten von Jugendlichen ganz oben, und das persönliche Geräteportfolio hat sich kontinuierlich erweitert. War es in den 1980ern noch eine kleine Sensation, endlich den eigenen Röhrenfernseher oder gar ein eigenes Telefon im Zimmer zu haben, ist heute ein Leben ohne Smartphone für viele kaum denkbar.

Mit Blick auf das eigene Geräteportfolio sind aber offenbar erste Sättigungseffekte erreicht. Jugendliche bekunden auf die Frage nach weiteren Wünschen für digitale Geräte eine hohe Zufriedenheit und können kaum Dinge nennen, die sie noch bräuchten - abgesehen von generellen Updates und neuen Geräteversionen. Dies gilt mitnichten nur für wohlhabendere Haushalte, sondern für Jugendliche aller sozialen Schichten. Auch andere Studien zum digitalen Familienalltag zeigen, dass die Ausstattung mit technischen Geräten keine Frage des Geldbeutels ist. ${ }^{2}$

2 Borgstedt, Silke et al. (2015): DIVSI U9-Studie Kinder in der digitalen Welt. Eine Grundlagenstudie des SINUS-Instituts Heidelberg im Auftrag des Deutschen Instituts für Vertrauen und Sicherheit im Internet (DIVSI). Abrufbar unter: https://www.divsi.de/wp-content/uploads/2015/06/U9-Studie-DIVSI-web.pdf 
Typische Zitate zur |llustration

( Ich brauche eigentlich nichts mehr, ich habe ja alles. Ein großer Fernseher zum Beispiel. Da überlegt man mal so drüber. Na das krieg' ich dann auch immer zu Weihnachten oder so was. Deswegen brauche ich eigentlich nichts mehr. (männlich, 15 Jahre, Adaptiv-Pragmatische)

Mein Laptop ist relativ neu. Freunde von mir haben zum Beispiel gar keinen Laptop, oder sie müssen sich den mit ihren Geschwistern teilen. Da bin ich echt gut dran. Und ein iPhone 5c ist auch nicht das schlechteste Handy. Mein Vater hat auch viel solches Zeugs. Er ist Online-Redakteur. Und deshalb sind wir in der Familie gut ausgestattet. (weiblich, 15 Jahre, Expeditive)

( Also von der Konsole her bin ich sehr zufrieden. Und mit meinem Smartphone bin ich normalerweise auch zufrieden. Aber da habe ich halt einen selbst fabrizierten Schaden dran. Und wahrscheinlich kommen daher auch die Macken ab und an. (männlich, 15 Jahre, Materialistische Hedonisten)

( ICh bin sehr zufrieden, weil ich habe ein Laptop. Klar man könnte jetzt auch sagen: ,Ja, du hast einen Standrechner mit übelsten Boxen und so.' Ich bin schon richtig froh, dass ich jetzt einen Laptop habe mit 14, weil ich kenne viele, die haben noch nicht mal ein Smartphone. (männlich, 14 Jahre, Materialistische Hedonisten)

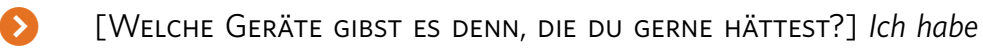
alles das, was ich brauche. Also, die meisten brauche ich alle gar nicht. (männlich, 14 Jahre, Prekäre)

\section{Mediennutzung = Internetnutzung}

Relevant sind im Hardware-Portfolio der Jugendlichen vor allem diejenigen Geräte, die Online-Anwendungen ermöglichen. Diese Option ist für Jugendliche selbstverständlich und unhinterfragt. Sie gehören zur ersten Generation von Jugendlichen in Deutschland, die das Internet nicht nur selbstverständlich nutzt, sondern online lebt.

Jugendliche nennen Fernseher nur selten als zentrale und wichtige Geräte im eigenen Portfolio, jedoch wird aus den weiteren Schilderungen 
des medialen Alltags deutlich, dass das TV dennoch vorhanden ist und genutzt wird. Es ist aber offenbar ein Medium, das sich bereits so selbstverständlich etabliert hat, dass es nicht mehr nennenswert ist, aber keines, dem man ungeteilte Aufmerksamkeit schenkt. Es gilt zudem als Mittel der Wahl zum „Abschalten“, wenn man mal einfach nur "abhängen" will oder als willkommene Alternative, wenn aus irgendwelchen Gründen kein Internet zur Verfügung steht.

Zur Spielekonsole gibt es eine deutlich stärkere emotionale Bindung als zum Fernseher. Sie kann allerdings immer weniger als Offline-Gerät eingeordnet werden. Jugendliche spielen hiermit vor allem online, zudem wird die Konsole häufig auch zum Anschauen von Videos im Internet verwendet.

Fernseher und Spielekonsole sind somit bei Jugendlichen weiterhin wichtig, reichen aber an die Bedeutung des Smartphones bei Weitem nicht heran.

(1) Das Gute wäre aber, dass ich [AN EINEM TAG OHNE HANDY] endlich Freizeit für einfach mal nur Fernsehen schauen habe, weil ich seit Monaten kein Fernsehen schaue, weil ich übers Internet alles mache. (männlich, 16 Jahre, Adaptiv-Pragmatische)

(1) [INT: GIBT ES GERÄTE, DIE DU GERNE NUTZEN WÜRDEST ABER NICHT HAST?] Eine PS4. Alle können darauf Filme schauen oder Musik hören. Und ich brauche dafür mein Handy oder muss über den Fernseher gucken. Also eine Xbox ist schon manchmal vorteilhaft. Und wenn ich mir dann ein, zwei Spiele hole, könnte ich mit denen auch online spielen. (weiblich, 16 Jahre, Materialistische Hedonisten)

\section{Das Handy bzw. das Smartphone ist das wichtigste technische Gerät für Jugendliche.}

Während der Fernseher bei Jugendlichen eher an die Peripherie der häuslichen Mediengalaxie gerückt ist, - zwar wichtig, aber im Hintergrund steht das Handy, das fast alle mittlerweile in der Smartphone-Variante 
besitzen, unangefochten an der Spitze der wichtigsten Dinge im Leben. Halb verständnislos, halb bewundernd bekunden 14- bis 17-Jährige noch nicht einmal zehn Jahre nachdem Smartphones auf dem deutschen Markt Einzug erhalten haben, wie schwierig es doch "damals" sein musste, die Tätigkeiten des Alltags ohne mobile, internetfähige Geräte zu bewältigen.

Typische Zitate zur Illustration
(7) Und ohne Handy, da hat man überhaupt keinen Kontakt zu anderen Menschen. Dann könnte man halt zehn Tage durchgehend etwas machen und etwas unternehmen. Aber dann muss man auch etwas ausmachen, und wenn man kein Smartphone hat, wird das alles ein bisschen schwer. (männlich, 15 Jahre, Adaptiv-Pragmatische)

(7) Füher sagt meine Schwester - sie ist zehn Jahre älter als ich, hat man noch in diesen Telefonzellen telefoniert. Überleg mal, wie krass krank das sein muss, dass man Münzen in diesen Automat reinsteckt. Ich weiß nicht, ich kann mir das nicht vorstellen. Ich kann's mir einfach nicht vorstellen, dass man kein Telefon in der Tasche hat. Stell dir vor, es passiert was oder mir ist langweilig auf der Straße. Was mache ich?! (weiblich, 16 Jahre, Adaptiv-Pragmatische)

(1) Es ist mir sehr wichtig, das Smartphone. Es ist wichtig. Wenn man ein Smartphone hat, dann lernt man Menschen kennen. Dann ist man nicht immer so alleine. Zum Beispiel bei Facebook. Jeder sieht jeden natürlich bei Facebook. Letztens habe ich meine Kindergartenfreunde gesehen und jetzt schreiben wir. (weiblich, 15 Jahre, Materialistische Hedonisten)

Längst sind Smartphones selbstverständliche Begleiter für alle Lebenslagen geworden. Gerade die "Alles-in-Einem"-Funktion wird von den Jugendlichen hervorgehoben. Für sie ist das Handy Infozentrale, Navi, Unterhaltungsmedium und - vor allem - Kommunikationsstandleitung zu den Freunden. Gerade die Integration diverser Funktionen macht das Smartphone nicht nur unentbehrlich, man hat vielmehr eine persönliche Beziehung mit emotionalen Qualitäten zu ihm aufgebaut. Jugendliche betonen, dass ihr Leben durch das Smartphone entspannter sei, da sie 


\section{Auszüge aus Hausaufgabenheften: \\ WAS IST FÜR DICH DAS WICHTIGSTE TECHNISCHE GERÄT UND WARUM?}

Mein Handy.

Mit einem Handy wache ich forst alles. Daher, dass ich auch hin Computs hab, ist mein Handy aich wein Comyltker. ich untze es fiur che Schule, Pricat und acch ads Ferubedie nung für unserem Feruselves.

männlich, 16 Jahre, Expeditive

Aufjedenfall mein Handy, weil ich es einfach

brauche!

Ich mache mit meinem Haudy sehr viel:

chatten, spielen, surfen, telefonieren, u.s.w.

weiblich, 14 Jahre, Konservativ-Bürgerliche

Mein Handy, weil ich dadrauf den ganzen Tag erreichbar bin. Und weil ich den ganzen Tag mit meinen Freundinnen chatten und tekfonieren kann.

weiblich, 15 Jahre,

Materialistische Hedonisten

Tir mich ist mein Compuler das wichtigite technische Gerat, da or no gut wic alle T-unhtionen vereint, die sonst nur einzelne technincle Gerate lesitzen.

Jch nutze den Compuler hauptiäclich für soziale Netzwerhe wie 2.B. Tacebook, aler auch um Sevien und Tilme zu nchouen. Außerdom höre ich vom Computer aus Musih. piele Spiele und mutze iln vor allem in der Schulzeit ah Informationsquelle.
Mein Saptop ist mir wichtig. Aber auch meine Ps4 und mein bandy Also Loptop, Pst und Handy Mein Laptop ist mir sehr wichtig, weil, ich meine "Geschichten" darauf schreibe "Wenn mein Laptop kaputt geht, sind meine ganzon Geschriebenen Dokumente weg lon sollte mir viellechl ein USB-Stick holen.....

Meine Ps4 ist mir auch sehr wichtig. Es gibt nicht cooleres als die Welt der Videospiele D th liebe sie cinfoch Mein Handy hat ein eigenen Namen: D. Er heißt Peter Jedoch habe ich ein neus Hardy hor paar Sagen bekommen und Peter dient zu náchst nur als wecker.

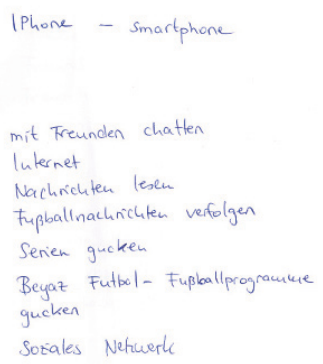

männlich, 15 Jahre, Konservativ-Bürgerliche

- Handy

- personen erréchen

- In Notfall benutzea

- Reitvertreib

männlich, 16 Jahre, Prekäre weiblich, 14 Jahre, Experimentalistische Hedonisten
Mein Handy. Ich bin überall erreichbar. Ich weiß immer wann und wo die nächste Bahn fährt. Es weckt mich. Es ist meine Uhr. Es ist ein mobiles Nachschlagewerk. Es unterhält mich. männlich, 17 Jahre, Adaptiv-Pragmatische

$$
\begin{aligned}
& \text { - Mein Kandy } 3 \\
& \text { - ich selreibe mit menen Freundar } \\
& \text { k Familie und halk eler } \\
& \text { whatsapp eine wirkich tolk } \\
& \text { Freundsceryft a lenschen ilarall } \\
& \text { aut der welt acfecht. } \\
& \text { Myrrthe - Nideriande } \\
& \text { Lisa - Halien ... ) } \\
& \text { - les macere Bilder } \\
& \text { - Lexe kurzgoceiceiten aut Wattpad } \\
& \text { - poste Bilder auf mstagram } \\
& \text { - tweete af Twitte } \\
& \text { - Hore Musik } \\
& \text { - "snape" mit Freunden al Snapchat }
\end{aligned}
$$


sich schneller auf den neuen Stand bringen könnten. Zudem fühlen sie sich mit dem Handy sicherer, wenn sie allein unterwegs sind.

Typische

Zitate zur

|llustration

( Man kann auch ohne Handy. Wir machen auch manchmal was ohne unsere Handys. Aber sonst, man muss erreichbar sein, das ist wichtig. Einer muss immer das Handy dabei haben, weil es könnte auch mal was passieren. (weiblich, 17 Jahre, Prekäre)

(7) Also ich habe es schon mal vergessen zuhause. Ich fand das relativ schlimm. Zum einen, weil ich hätte ja wichtige Nachrichten über WhatsApp kriegen können, was natürlich nicht der Fall ist, oder wenn ich jetzt zum Beispiel meine Bahn verpasse, meine Eltern anrufen muss. (männlich, 14 Jahre, Materialistische Hedonisten)

\section{WhatsApp, Instagram \& Co sind unverzichtbare Infrastruktur für soziale Teilhabe.}

Zentrale Funktion digitaler Medien ist für Jugendliche die Pflege und Aufrechterhaltung von Freundschaften. Entgegen weitläufiger Meinungen, dass Medien zu einer Verarmung der sozialen Beziehungen führen, haben Jugendliche selbst eher das Gefühl, ohne Medien sozial zu verarmen. Dass Jugendliche ganze Nächte allein im Zimmer mit Ego-Shootern verbringen, ist offenbar nur eine Facette intensiver Mediennutzung, bzw. ein Klischee. Für den Großteil der Jugendlichen geht es vorrangig um sozialen Austausch, d. h. Informationsabgleich, Posten von Links, Musik und Verabredungen, die dann wiederum offline stattfinden. Im Unterschied zur SINUS-Jugendstudie 2012 zeigen sich hierbei kaum mehr Unterschiede zwischen den Lebenswelten. Jugendliche, die im Internet nur zuschauen, was die anderen machen, aber sich selbst nicht einbringen, findet man auch in den prekären Lebenswelt kaum noch.

Typische Zitate zur Illustration

Eine ziemlich gute Freundin von mir wohnt zum Beispiel am Ende der Welt irgendwo in Halle. Das ist ganz weit weg. Sie braucht eine dreiviertel Stunde hierher. Mit ihr schreibe ich relativ viel. Manchmal sehen wir uns auch am Wochenende. Aber wir halten vor allem über das 
Handy Kontakt. Wenn wir das nicht hätten, würden wir uns vielleicht nur zweimal im Monat sehen. Ich glaube, unsere Beziehung wäre dann ganz anders. (weiblich, 15 Jahre, Expeditive)

(1) Das wichtigste technische Gerät ist mein Handy, weil ich mit meinem Handy mit jedem, der auch ein Handy hat, in Kontakt bleiben kann. Also wenn was ist, kann ich anrufen oder die mich. Oder wenn mal einem langweilig ist, dann kann man einfach schreiben, man kann Fotos schicken und das finde ich wichtig. (weiblich, 17 Jahre, Materialistische Hedonisten)

I Ich kommuniziere hauptsächlich mit Freunden über WhatsApp und alles Mögliche. So soziale Dinger, Facebook, Instagram und YouTube-Videos gucke ich viel. (männlich, 15 Jahre, Adaptiv-Pragmatische)

(7) WhatsApp ist sozusagen das Wichtigste auf meinem Handy. Darüber wird alles besprochen und geplant. Ansonsten nutze ich noch Instagram und Snapchat. Das nutze ich auch eigentlich fast täglich. (weiblich, 17 Jahre, Expeditive)

(7) Und auf Instagram bin ich eigentlich auch jeden Tag. Ich würde sagen, ich bin stundenlang auf Instagram. (weiblich, 17 Jahre, Konservativ-Bürgerliche)

Dass Vernetzung eine immer größere Rolle bei der Nutzung digitaler Medien spielt, liegt auch in der Weiterentwicklung der Online-Angebote begründet. Ging es bei neuen Produkten vor ca. zehn Jahren eher um neue Geräte und neue mobile Online-Angebote (Apps), so zeichnet sich der aktuelle Innovationsschub vor allem durch die Ergänzung um Vernetzungsoptionen bestehender Angebote aus. War Interaktion vor Jahren auf das Senden und Empfangen von Mails, Posts und die Teilnahme in Chat-Foren beschränkt, so hat sich der Community-Charakter zahlreicher Online-Dienste (jenseits "klassischer" Online-Communities wie Facebook) in den letzten Jahren verstärkt. Unter anderem werden die für Jugendlichen wichtigen Foto- und Videoplattformen zu Online-Gemeinschaften (z.B. YouTube, Instagram) und die bekanntesten User unter ihnen zu Stars. 


\section{FOMO - fear of missing out}

Die andere Seite der Medaille ist, dass es vielen schwer fällt, sich - digital - voneinander zu trennen. Wenn sich keiner mehr ausloggt, Apps schließt oder das Gerät ausschaltet, bleibt man dauerhaft verbunden und ist in permanenter Kommunikationsbereitschaft. Die Angst, etwas zu verpassen, ist empirische Realität bei Jugendlichen in Deutschland.

[INT: WIE WÄRE EIN TAg OHNE DEIN SMARTPHONE?] Mies. Ich gucke immer gerne, hat mir jetzt der und der geschrieben oder nicht? Und wenn man dann wei $\beta$, ja, der hat es gelesen, aber schreibt nicht zurück, Arsch! Und wenn das nicht da wäre, ich die ganze Zeit so: Handy, ich will es wieder haben, bitte, gib mir das Handy! Ich hatte mal sechs Stunden keinen Empfang und habe die ganze Zeit gedacht: Empfang, bitte komm wieder! (weiblich, 14 Jahre, Experimentalistische Hedonisten)

(7) Es ist in der heutigen Zeit eigentlich fast nicht mehr wegzudenken, weil viele sich auf die Smartphones an sich beschränken und den Kontakt eigentlich fast nur noch per Nachrichtendienst halten können. Ich kenne keinen, der jetzt wirklich noch als junger Mensch so Briefe schreibt. Das dauert alles viel zu lange. (männlich, 16 Jahre, Adaptiv-Pragmatische)

( Also wenn ich dann mal doch bis um zwölf oder noch länger wach bin, liegt das meistens daran, dass mein Handy mich nicht in Ruhe lässt. Also das ist dann die ganze Zeit in Beschäftigung. (männlich, 15 Jahre, Materialistische Hedonisten)

Allerdings haben sich auch hier bereits einige Verhaltens-Kodizes gebildet: So gelten direkte Anrufe mittlerweile als verpönt, da man nicht weiß, ob man jemanden gerade stört. Texten hingegen geht immer und ist entweder Ankündigung eines Telefonats oder - weit häufiger - die alleinige Form sich auszutauschen oder zu verabreden.

(7) Wenn man anruft, weiß man nie, ob man den anderen stört. (weiblich, 15 Jahre, Expeditive) 
(1) Ohne Smartphone? Ich glaube, dann würde ich den ganzen Tag nur zu Hause herumhängen und keinen Kontakt zu meinen Freunden haben. So über Festnetz oder so telefoniert man ja einfach nicht mehr. (männlich, 17 Jahre, Experimentalistische Hedonisten)

( Ich benutze viel Facebook, so Nachrichten, WhatsApp, Snapchat, Instagram und zum Telefonieren auch ab und zu, aber gar nicht so oft. (weiblich, 17 Jahre, Adaptiv-Pragmatische)

(8) Telefonieren tue ich nur mit meinen Eltern und bloß Festnetz, wenn überhaupt. (männlich, 14 Jahre, Konservativ-Bürgerliche)

Gar nicht mit anderen Jugendlichen online vernetzt zu sein, ist ungewöhnlich und wird mit Außenseitertum gleichgesetzt. Wer online nicht dabei ist, ist auch sonst "draußen“. Digitale Teilhabe wird somit zur sozialen Teilhabe.

(1) [INT: ECHT, WIE WÄre SO EIN TAG OHNE HANDY FÜR DICH?] Nicht besonders gut. Sagen wir es mal so. [INT: WIESO NICHT?] Man weiß nicht, was die Freunde machen, man bekommt eigentlich so wirklich gar nichts mit. (männlich, 15 Jahre, Adaptiv-Pragmatische)

( Ich muss sagen, seitdem ich bei WhatsApp bin, ist es so das, worüber man alles macht. Also man schreibt über WhatsApp, man verabredet sich über WhatsApp. Also ganz viel eigentlich am Tag. Aber das nervt mich ehrlich gesagt, weil alles geht über WhatsApp nur noch heutzutage. Und ich fände es eigentlich schön, wenn man mal wieder telefonieren würde. Nicht nur dieses Schreiben immer. Das nervt mich eigentlich. Aber es geht alles darüber. Und entweder man passt sich an oder man wird ausgeschlossen sozusagen. (weiblich, 16 Jahre, Sozialökologische)

\section{Jugendliche sind in sozialen Situationen auch genervt von Smartphones.}

Erstmalig zeigen sich jedoch ebenso negative Aspekte des unbegrenzten mobilen Verfügbarkeits-Modus. Gerade in sozialen Settings (Party, 
gemeinsam beisammen sitzen o.ä.) können Handys auch nerven. Permanentes "Starren aufs Display" und "dauerndes Herumgetippe" gelten bei manchen Jugendlichen mittlerweile als uncool und vermeidbar (insbesondere in der Konservativ-bürgerlichen und Sozialökologischen Lebenswelt).

Typische Zitate zur Illustration
(1) Ich glaube, das wird irgendwann so sein, dass es wirklich in Klubs Schilder gibt: "Handys abgeben" oder "Handys daheim lassen. Hier drin wird sich richtig unterhalten" oder sowas. Das ist ja auch in Cafés so. Das sieht man auch bei uns im Verein. Wir sitzen am runden Tisch und jeder hat sein Handy vor der Nase. Und das ist schon irgendwie doof. (weiblich, 17 Jahre, Konservativ-Bürgerliche)

(7) Wir haben auch eine bei uns, die ist, ich will jetzt nicht sagen Facebook-süchtig, aber sehr von Facebook abhängig, und die ist fast jede Minute darauf. (weiblich, 15 Jahre, Konservativ-Bürgerliche)

(7) Mich stört es manchmal, wenn sie nur am Handy hängen oder nur am Zocken sind. Manchmal will man auch reden, das stört dann, aber ist halt so. (weiblich, 16 Jahre, Experimentalistische Hedonisten)

Souveränität im Umgang mit digitalen Medien zeigt sich aus Perspektive der Jugendlichen auch daran, Geräte gezielt und angemessen einzusetzen und vor allem daran, ob man in der Situation wirklich etwas mit anderen zu kommunizieren hat, das wichtiger als das aktuelle Real Life-Geschehen ist. Es gilt - insbesondere in bildungshöheren Lebenswelten - als Kompetenz und Distinktionsmerkmal, Geräte auch mal ausschalten zu können. In diesem Zusammenhang greifen Jugendliche häufig das Thema "Mediensucht" auf. Von denen, die "süchtig" sind, möchte man sich deutlich abgrenzen („,in meiner Klasse sind 50 \% süchtig. Man sollte es halt echt dosieren"). Bittet man die Jugendlichen, selbst Fragebögen zum Thema "Ich im Internet" zu erstellen, ist die Frage nach der Abhängigkeit von digitalen Medien entsprechend relevant, wie die folgende Abbildung zeigt.

Nicht ohne Selbstkritik wird trotz aller bekundeten Bemühungen um einen "maßvollen" Umgang beklagt, wie groß der Stellenwert des eigenen Smartphones ist - gerade in der Funktion als soziales Back-Up. Ein Leben ohne Handy ist folglich zwar vorstellbar, aber nur unter der 
Bedingung, dass alle anderen auch darauf verzichten oder(monetäre) Kompensationen angeboten werden.

Interviewerin: weiblich, 16 Jahre, Adaptiv-Pragmatische

WÜRDEST DU FÜR $200 €$ ZWEI WOCHEN LANG AUF DEIN HANDY UND IPAD VERZICHTEN KÖNNEN?

I Ich glaube, wenn ich ehrgeizig bin, und ich will doch eigentlich jetzt für meinen Führerschein sparen, dann würde ich das vielleicht zwei Wochen durchziehen. Aber ansonsten ...

Auszüge aus Gesprächen zwischen Jugendlichen zum Thema

\section{KANNST DU ES DIR DENN VORSTELLEN?}

( Ich weiß nicht. Also für $200 €$ würde man sich das überlegen, aber durchhalten ... Aber zwei Wochen finde ich schon, dass das voll die krass lange Zeit ist. Wie gesagt, ich stehe mit meinem Handywecker auf, bis ich in die Schule gehe, bin ich die ganze Zeit im Internet drin. Ich habe sogar mir- du ja auch - so einen Ersatzakku gekauft, falls es ausgeht.

Auszüge aus Fragebögen, die Jugendliche zum Thema "Ich im Internet" entwickelt haben.

\section{bist du schon sichtig?}

weiblich, 14 Jahre, Materialistische Hedonistin

Ich im Internet

- Könntest du 1 Woche ohne Internet auskommen?

- In wie vielen Aktiven Whatsapp Gruppen bist du derzeit?

männlich, 17 Jahre, Konservativ-Bürgerliche

Ich im Internet:

Durch WLAN und mobiles Internet sind wir jeden Tag online und nahezu immer erreichbar. Wozu benutzt du das Internet regelmäßig?

Wie abhängig vom Handy würdest du dich einschätzen?

Erleichtert das Handy das Leben? Oder macht es auch zusätzlichen Druck?

weiblich, 17 Jahre, Expeditive 


\title{
4.2 Aushandlungsprozesse im digitalen Raum
}

\begin{abstract}
"Always on" zu sein ist normal - und wird auch von den Eltern weitgehend akzeptiert.
\end{abstract}

Nur drei Prozent der 14- bis 17-Jährigen in Deutschland nutzen das Internet gar nicht. Zum Vergleich: In der Gesamtbevölkerung gehörten 2014 noch rund 23\% zu den Offlinern. Die Online-Zeiten Jugendlicher haben sich zudem intensiviert, was zum einen durch die Ergänzung von Geräten um Online-Funktionen bedingt ist, aber auch durch flächendeckende Versorgung mit Flatrate-Angeboten. Eine eher neue Entwicklung ist daher der Wegfall zeitlicher Beschränkungen durch ökonomische Argumente seitens der Eltern. Die leere Prepaid-Card, die von den Eltern nur als Belohnung für Haushaltsmithilfe oder zum Geburtstag wieder aufgeladen wird, gehört zunehmend der Vergangenheit an.

Online- und Offline-Zeiten sind somit für Jugendliche fließend und nicht immer klar voneinander zu trennen, insbesondere weil die Geräte in der Regel nicht ausgeschaltet werden. Online-Sein wird kaum noch als technisch hergestellte Verbindung betrachtet, niemand "geht ins Internet". Online zu sein gleicht eher einer Situationsbeschreibung, dem Normalzustand. Es ist das quasi-natürliche Zugriffspotenzial auf eine Vielzahl von Daten in Echtzeit und die gleichzeitige Empfangsbereitschaft für eintreffende Daten, die als persönlich relevante Informationen eingeordnet werden. $^{3}$

Der Umgang mit und das Sprechen über "das Internet" ist folglich weitgehend entdramatisiert. Euphorische Online-Begeisterung ist aus Perspektive der Jugendlichen eher Sache der Älteren oder der "Online-Neulinge". Für sie ist das Internet lediglich praktisch und die einfachste Lösung für Fragen und Probleme im Alltag. Wie Strom und Wasser ist WLAN die Grundversorgung, um mit der Welt verbunden zu sein.

3 Borgstedt, Silke et al. (2014): DIVSI U25-Studie. Kinder, Jugendliche und junge Erwachsene in der digitalen Welt. Eine Grundlagenstudie des SINUS-Instituts im Auftrag des Deutschen Instituts für Vertrauen und Sicherheit im Internet. Abrufbar unter: https://www.divsi.de/wp-content/uploads/2014/02/DIVSI-U25-Studie.pdf 
(1) Also ich lass es immer an, aber ich mache es auf lautlos. (weiblich, 17 Jahre, Materialistische Hedonisten)

( Ich habe einen Vertrag. Ich weiß gerade gar nicht, wie der heißt. Der ist so für Jugendliche. 25 Euro mit 3GB Internet, SMS Flat und Telefonie Flat. Ja, reicht. (weiblich, 17 Jahre, Konservativ-Bürgerliche)

( Ich habe eine Prepaidkarte. Aber ich habe mir eine Internet-Flat dazu gebucht, damit ich immer Internet habe. (weiblich, 14 Jahre, Adaptiv-Pragmatische)

( Ich habe eine Allnet-Flat, also SMS, Anrufe und Internet. (männlich, 15 Jahre, Materialistische Hedonisten)

( [INT: ABER WÜRDEST dU DENN SAgEN, DU KANNST OHNE DEIN HANDY ODER DEINEN COMPUTER LEBEN?] Ich könnte bestimmt, aber es wäre kein schönes Leben mehr. [INT: ABER WÜRDEST DU AUCH SAGEN, DASS ES SELBSTVERSTÄNDLICH IST, ES ZU BESITZEN ODER ES ZU HABEN?] AlsO es hat zwar jeder aus meinem Freundeskreis und generell jeder aus meiner Schule ein Smartphone, aber es liegt natürlich auch extrem an den europäischen Umständen. Also jetzt beispielsweise, wenn wir irgendwie in Ghana oder so leben würden, das wäre was ganz anderes. Und für die ist es ja eine komplett andere Welt. Deswegen ist es ja auf jeden Fall nicht selbstverständlich, aber in Deutschland ist es schon selbstverständlich. (weiblich, 17 Jahre, Experimentalistische Hedonisten)

\section{Internet ist ein Raum der Möglichkeiten - weitgehend befreit vom Eingriff durch Erwachsene.}

Das Online-Verhalten verläuft bei den 14- bis 17-Jährigen überwiegend in Eigenregie. Die Eltern lassen nach Bekunden der Jugendlichen weitestgehend alle Freiheiten im Netz. Es gibt teilweise Regeln für bestimmte Situationen (z. B. kein Handy beim Essen oder während der Schulzeit) oder seitens der Eltern wird angemerkt, dass insbesondere das Smartphone deutlich zu lang in Verwendung sei, vor allem am späten Abend bzw. in der Nacht. Weniger geht es dabei aber um die Online-Aktivitäten und -inhalte selbst, als um den verpassten Schlaf, wenn am nächsten 
Tag Schule ist. Einzige Regel ist häufig, dass Downloads aller Art (insbesondere Filme und Musik) verboten sind. Hierbei wird seitens der Eltern nicht zwischen legalen und illegalen Downloads unterschieden, da sie selbst nicht durchschauen, was eigentlich erlaubt ist und was nicht. Sicherheitshalber wird dann jegliche Art von Downloads untersagt. Bilder werden laut Jugendlichen hingegen von den Eltern als "ok" eingestuft. Von Eltern erhalten Jugendliche auch den Hinweis, dass sie darauf achten sollen, welche Informationen sie von sich im Netz präsentierten. Eigens hergestellter Content, wie z. B. selbstgedrehte Filme spielen aus Perspektive der Jugendliche ohnehin nur eine kleinere Rolle, sie sehen sich vorwiegend als Rezipienten, die den Hauptnutzen daraus ziehen, bereits verfügbare Inhalte im Freundeskreis entsprechend zu verteilen.

Typische Zitate zur Illustration

(7) Höchstens wenn es mal zu spät wird. Dann sagt Mama: "Jetzt reicht es dann aber mal." Und dann lasse ich es aus. (weiblich, 16 Jahre, Sozialökologische)

(7) Eigentlich überwacht sie das gar nicht. Sie denkt, glaube ich, ich weiß schon, was ich mache. (weiblich, 15 Jahre, Adaptiv-Pragmatische)

(7) Außer wenn wir am Tisch sind. Da müssen wir unsere Handys weglegen, weil das meine Mama total stört. (weiblich, 14 Jahre, Adaptiv-Pragmatische)

Früher gab es mal Regeln am Computer, also wegen gewissen Internetseiten. Aber ansonsten gibt es eigentlich keine Regeln. (männlich, 16 Jahre, Expeditive)

(1) Ich habe doch eine Regel: Ich darf nichts downloaden. Also wenn man eine Website nutzt, nichts downloaden. Keine Filme, keine Links. Bilder sind okay, wenn man das downloaded, aber niemals Filme, die darf ich nicht downloaden. (weiblich, 17 Jahre, Adaptiv-Pragmatische) 


\section{Mediennutzung ist kein großes Streitthema, da viele Eltern mittlerweile selbst versierte Onliner sind.}

Das Verhältnis zu den Eltern wird mit Blick auf die Mediennutzung insgesamt als entspannt beschrieben und ist kaum von großen Konflikten, sondern durch einen vertrauensvollen Umgang miteinander gekennzeichnet. Auch andere Befunde deuten darauf hin, dass sich das Verhältnis zu den Eltern in punkto Internet verändert hat. Standen vor einigen Jahren noch Auseinandersetzungen zum „wieviel“ und "was" auf der Tagesordnung, ist seitens der Eltern eher eine tolerierende bis unterstützende Haltung zu beobachten. Wie andere Untersuchungen zeigen, beobachten auch Eltern eine zunehmende Bedeutung digitaler Medien für die gesellschaftliche Teilhabe und den beruflichen Erfolg ihrer Kinder - unabhängig davon, wie sie dies persönlich bewerten. Bereits die Ausstattung mit digitalen Medien dient dabei wahlweise als bewusste Bildungsinvestition oder als offensives Zeichen, "mithalten“ zu können. Nicht selten haben Jugendliche zudem ihr erstes Handy von den Eltern geschenkt bekommen, um für sie erreichbar zu sein.

(7) Also meine Mutti, die ist da nicht so streng, die ist da eigentlich total locker, was das angeht. Nur es ist natürlich so, wenn ich jetzt gerade beim Abendbrot sitze oder frühstücke oder was auch immer und ich

Typische Zitate zur Illustration dann am Handy bin, dann sagt sie schon mal "Du isst jetzt, kannst du das weglegen?". Ist auch eigentlich nie so, dass ich das mache. (weiblich, 14 Jahre, Konservativ-Bürgerliche)

( Ich glaube, meine Eltern sind da echt tolerant. Es gibt zum Beispiel Freunde von mir, die am Tag nur eine halbe Stunde ins Internet dürfen. Ich darf unbegrenzt hinein. Meine Mutter sagt zwar: „Jetzt mach mal aus". Aber sie würde es mir auch nie direkt verbieten oder wegnehmen. (weiblich, 15 Jahre, Expeditive)

Wir haben Zeiten. Also nachts oder abends muss ich mein Handy in die Küche legen, weil ich sonst einfach nicht schlafen würde. Ich würde ansonsten die ganze Nacht am Handy sein. Und deswegen 
ist es schon besser, wenn ich es unten hinlege. (männlich, 15 Jahre, Sozialökologische)

Gerade bei Konservativ-bürgerlichen Jugendlichen, bei Expeditiven und Sozialökologischen gibt es ein hohes Maß an Selbstregulationswunsch und -kompetenz - wie auch bereits das vorausgehende Unterkapitel zeigte. „Ausschalten können“ ist wichtig und ein Distinktionsmerkmal zu den "Dauerdaddlern“. Auch wenn man es im Alltag nicht immer hinbekommt, zeigt man doch gewisse Bemühungen. In den Lebenswelten der Materialistisch-hedonistischen Jugendlichen und der Prekären gibt es deutlich häufiger Auseinandersetzungen mit den Eltern, wenn es um die Nutzungsdauer digitaler Medien geht. Die von den Eltern aufgestellten Regeln werden zumeist als willkürlich und nicht plausibel empfunden. Zudem haben die Jugendlichen in allen - aber insbesondere diesen - Lebenswelten diverse Strategien entworfen, die Regeln der Eltern zu umgehen (z. B. sich morgens den Wecker stellen, bevor die Eltern aufstehen, um die letzte Online-Aktivität bei WhatsApp in den frühen Morgen statt in die späte Nacht datieren zu können).

(7) Auch wenn meine Eltern sagen würden, "Du darfst nur von dieser Uhrzeit auf diese Uhrzeit ans Handy", würde ich trotzdem an meinem Handy sein. Nur nachts, wenn ich bis drei/vier Uhr noch online bin, dann sagen meine Eltern, ich soll schlafen, aber mache ich nicht. (weiblich, 14 Jahre, Prekäre) 


\subsection{Digitale Medienkompetenz}

\section{Jugendliche wissen um digitale Medienkompetenz als zentrale Voraussetzung für gesellschaftliche Teilhabe.}

Da Jugendliche fest von der fortschreitenden Digitalisierung überzeugt sind, steht es für sie außer Frage, dass man zumindest über ein Basiswissen im Umgang mit digitalen Medien verfügen muss, um künftig Teilhabe sicherstellen zu können - vor allem auch mit Blick auf den Beruf.

Entscheidend für die Einschätzung der Bedeutung digitaler Medienkompetenz ist der Digitalisierungsgrad des Elternhauses. In Elternhäusern der postmodernen Lebenswelten gilt Medienkompetenz mittlerweile als Schlüsselkompetenz für den beruflichen Erfolg der Kinder und wird in den privaten Bildungskanon integriert. ${ }^{4}$ Wenn Eltern selbst das Internet kaum beruflich nutzen, möchten Jugendliche in bürgerlichen Lebenswelten dies auf jeden Fall anders machen ("das kann man sich heute nicht mehr leisten“). In hedonistischen und prekären Lebenswelten üben Eltern seltener Berufe aus, in denen digitale Medien eine vorrangige Rolle spielen, hier übernehmen Jugendliche tendenziell die Vorstellung, dass digitale Medien und insbesondere das Internet beruflich für sie vermutlich keine große Rolle spielen werden.

( J Ja, ich finde es schon wichtig. Also zumindest in unserer Generation, jetzt zum Beispiel bei meinen Eltern oder so verstehe ich das ja noch, weil die sind ja nicht damit aufgewachsen. Aber von uns wird das ja so erwartet, weil wir das ja einfach schon von Anfang an so kennen. Also einerseits ist es auch irgendwie traurig, aber andererseits ist es auch gut, weil es schon hilfreich ist. (weiblich, 17 Jahre, Sozialökologische)

( Digitale Medien werden auf jeden Fall eine große Rolle spielen, weil sie weltweit eine große Rolle spielen. Es geht nicht anders. (männlich, 17 Jahre, Experimentalistische Hedonisten)

$4 \quad$ Borgstedt, Silke v. a. (2015): DIVSI U9-Studie Kinder in der digitalen Welt. Eine Grundlagenstudie des SINUS-Instituts Heidelberg im Auftrag des Deutschen Instituts für Vertrauen und Sicherheit im Internet (DIVSI). Abrufbar unter: https://www.divsi.de/wp-content/uploads/2015/06/U9-Studie-DIVSI-web.pdf

Typische Zitate zur |llustration 
( Im Job wird es auch immer wichtiger, dass man mit Technik umgehen kann. Doch, das bleibt auf jeden Fall. (weiblich, 17 Jahre, Expeditive)

( Technik findet man ja fast schon in jedem Beruf. Auch als Sekretärin, anstatt irgendwas noch zu schreiben, damals hat man das auch mit der Schreibmaschine gemacht, jetzt macht man das auch schon im PC. (weiblich, 15 Jahre, Sozialökologische)

( Ich glaube, sogar sehr wichtig. Weil die Zukunft wird ja jetzt immer mehr digitalisiert. Da ist dann auch immer wichtiger. Industrie, glaube ich. Es gibt zum Beispiel jetzt ein Studium in Industrie 4.0, also dann wird die Industrie einfach digitalisiert. Schon krass. (männlich, 16 Jahre, Materialistische Hedonisten)

In den Schilderungen der Jugendlichen gibt es ganz klare Unterschiede des Digitalisierungsgrades in den Berufen: Wer einen Bürojob hat, kommt am Internet nicht vorbei, weniger wichtig ist es allerdings für Automechaniker, Friseure und Hebammen. Ob digitale Medien somit später im Alltag zentral sind, hängt aus Sicht der Jugendlichen auch vom gewählten Beruf $a b$.

( Kommt natürlich darauf an, was man für einen Beruf nimmt. Wenn ich natürlich jetzt Handwerker bin, hat das natürlich jetzt nicht so eine große Rolle, außer ich will jetzt, Vogelhaus' googlen. Ansonsten, wenn man am Rechner arbeitet schon. Deswegen würde ich mir auch wünschen, weil es so viele Büro-Jobs gibt, dass man das mehr im Unterricht macht, aber macht man halt nicht. (männlich, 14 Jahre, Materialistische Hedonisten)

\section{Medienkompetenz erwerben Jugendliche „by doing“.}

Mit digitalen Medien umgehen zu können ist für Jugendliche kein bewusster Lernprozess, sondern ein kontinuierliches Hineinwachsen und Ausprobieren. Viele der Jugendlichen hat daher die Frage, wie sie sich ihre alltägliche Medienkompetenz aneignen, verwundert: Mit Medien muss man sich aus ihrer Sicht heute einfach auskennen. Das trifft besonders auf das technische Handling und die Bedienung von Interfaces der 
"Standardgeräte" zu: Handy/Smartphone, Tablets und Spielekonsolen. Es gilt als Selbstverständlichkeit, sich den Umgang mit diesen Geräten über "learning by doing" in Eigenregie beizubringen. Neben "trial and error" nutzen einige wenige auch Online-Tutorials oder Hilfe-Foren, wenn sie nicht weiter wissen oder etwas grundlegend lernen möchten. Meist probiert man es aber zunächst einfach selbst aus.

Auch der Umgang mit den gängigen Online-Diensten und Apps wird "automatisch" erlernt, frei nach dem Motto: draufklicken und schauen, was passiert. Das Internet gilt als "einfach" und selbsterklärend, zudem erhält man im Internet selbst Auskunft, falls man nicht mehr weiter weiß.

( Man muss andere nicht fragen, es gibt da so eine Lupe und mit der kann man alles durchsuchen, wenn man etwas finden will. (weiblich, 15 Jahre, Adaptiv-Pragmatische)

Typische Zitate zur Illustration

Probieren geht über Studieren, man probiert sich halt durch und dann irgendwann kannst du's halt. Hast du halt total den Durchblick. Ich habe mir eine App runter geladen zum Video schneiden, habe damit das Video so paar Schnitte an sich gesetzt und dann alles halt okay. (männlich, 16 Jahre, Adaptiv-Pragmatische)

Ich mach so Fanvideos, zum Beispiel jetzt zu meiner Lieblingsserie oder zu bestimmten Musikern mit Musik im Hintergrund. Dass man halt so Filmausschnitte da reinbringt und so was. Da gibt es ja viele. [INT: Wo KANNST DU SOLCHE SACHEN her?] Vieles von YouTube. Also ich habe vieles mir eigentlich selber beigebracht. Aber es gibt natürlich auch Videos, die zeigen, wie man das macht. (weiblich, 16 Jahre, Experimentalistische Hedonisten)

I Ich mach das ja nicht professionell, also ich habe da so Apps und dann probiere ich das halt aus und klatsche irgendeinen Filter oder einen Effekt drauf. Und dann schneide ich es zusammen wie es mir passt. (weiblich, 17 Jahre, Sozialökologische)

[INT: WIE HAST DU DIR DAS DENN ALLES ANGEEIGNET?] Das ist wirklich eine gute Frage. Das ist einfach so gekommen. Man weiß es gar nicht so genau. Ich glaube, weil man vielleicht einfach damit aufgewachsen ist 
und immer schon seit man klein ist, mal hier was machen musste, mal da, mal auf einer Spieleseite war. Da lernt man das dann einfach, wie man so etwas bedient. Aber direkt gelernt nicht. Das ist einfach so ein Prozess und irgendwann kann man es einfach. (weiblich, 16 Jahre, Sozialökologische)

I Ich habe ja auch keinen Ansprechpartner dafür so richtig. Sondern einfach googeln, wenn man was wissen will und dann findet man meistens auch eine Antwort. (männlich, 17 Jahre, Adaptiv-Pragmatische)

( Das Internet ist nicht so schwer. Das kann sich eigentlich jeder selber beibringen. (weiblich, 17 Jahre, Adaptiv-Pragmatische)

(2) Weiß nicht. Damals war ich schon immer so auf Kinderseiten so Toggo. de. Und dann wurde mir erklärt, das ist die Suchleiste, da kannst du was eingeben und was suchen. (weiblich, 15 Jahre, Sozialökologische)

\section{Eltern sind wichtige Ansprechpartner.}

Eltern sind durchaus Ansprechpartner bei technischen Problemen oder Fragen zum Internet, insbesondere wenn Freunde nicht weiterwissen oder man sich für Dinge jenseits der Peergroup-Themen interessiert. In der Forschung zur Mediensozialisation wird in letzter Zeit häufiger postuliert, dass Medienkompetenz innerhalb von Peergruppen kultiviert und transformiert werde und sich der generationenlogische Prozess damit auflöse - also die Tatsache, dass Wissen von einer zur nächsten Generation weitergegeben wird. Die Ergebnisse zeigen aber vielmehr, dass sich diese Beobachtung nicht verallgemeinern lässt und allenfalls für den sprunghaften Beginn der digitalen Entwicklung bzw. des mobilen Internets gilt (2005-2009). Die aktuelle Elterngeneration ist in weiten Teilen mittlerweile selbst digital kompetent, da sie bereits mit digitalen Medien aufgewachsen oder durch den Beruf entsprechend sozialisiert wurde und diese ähnlich wie die eigenen Kinder mit einer gewissen Selbstverständlichkeit betrachtet.

Zwar betonen aktuelle Publikationen zur Mediensozialisation, dass sich Medienkompetenz und Nutzungsmuster heute vorrangig ohne den Einfluss von Erwachsenen und vor allem häufig ohne das Zutun von 
Bildungsinstitutionen entwickeln. Jugendliche verfügen aber durchaus über ein ausgeklügeltes Unterstützungsnetzwerk in Technikfragen, wobei Familienmitglieder auch eine relevante Rolle spielen - selbst wenn Freunde zumeist erste Anlaufstelle für Fragen rund um digitale Medien sind.

Ich habe den Umgang mit dem Computer eher von meiner Mutter gelernt. Und Streaming und so etwas habe ich von meiner Stiefschwester gelernt. Die ist älter als ich. Oder von anderen Freunden. Die wissen, wo man etwas schauen kann, wo es sozusagen am ungefährlichsten ist. (weiblich, 17 Jahre, Adaptiv-Pragmatische)

( Also zum Beispiel Facebook, meine Mutter hat Facebook gehabt, habe ich gesehen, als ich klein war. Ich durfte mir eigentlich kein Facebook machen, ich habe trotzdem Facebook gemacht vor drei Jahren. (weiblich, 14 Jahre, Prekäre)

( Also wo ich das lerne? Also eigentlich weiß ich schon relativ viel. Bei meinen Freunden auch, ja, eigentlich nur bei meinen Freunden. (männlich, 15 Jahre, Materialistische Hedonisten)

( Ich habe das alles eigentlich selber ausgecheckt. Ich habe draufgeklickt und geschaut, was da so passiert. Und eigentlich hauptsächlich der Freund von meiner Mom. Der ist so ein Computerfreak. Der checkt das alles voll aus. (weiblich, 15 Jahre, Experimentalistische Hedonisten)

( Und wenn ich dann irgendwelche Fragen habe, wie ich das und das mache oder wie ich eine PowerPoint Präsentation gestalte am besten, dann gehe ich zu meinem Bruder. (weiblich, 16 Jahre, Konservativ-Bürgerliche)

Die meisten Sachen am Computer hat mir eigentlich mein Vater erklärt. Und bei meinem Handy habe ich das eigentlich alles selbst rausgefunden. Also so durch ausprobieren. (weiblich, 14 Jahre, Sozialökologische) 


\section{Anwendung gängiger Dienste und Selbstschutz im Netz gelten als zentrale Medienkompetenzen.}

Basis-Wissen im Bereich digitaler Medien ist aus Sicht der Jugendlichen die Kenntnis und das Bedienen einschlägiger Anwendungen (insbesondere Apps) sowie die Fähigkeit, sich generell auf Benutzeroberflächen zurecht zu finden. Teilweise werden Standards im Bereich Geschicklichkeit genannt (mit dem Daumen texten, Steuerungstechniken beim Gaming); diese dienen in der Peergroup zum Teil als Indikator dafür, wer besonders versiert ist und wer nicht.

Typische

Zitate zur

|llustration
Digitale Medien? Man sollte wissen, wie es funktioniert. Ich glaube, da würde es schon ausreichen, wie man es anschaltet und ausschaltet und wie man darüber überhaupt etwas öffnet, zu macht usw. Das würde glaube ich das Wichtigste sein, bei digitalen Medien. (männlich, 16 Jahre, Materialistische Hedonisten)

( Beim Handy, dass man mit den Tasten so schreiben kann, dass man damit umgehen kann. Wenn ich die Generation von meinen Eltern anschaue, wenn die dann immer so tippen mit ihrem Finger, das macht ja keiner bei uns. Mit dem Daumen schreiben können. Und dass man weiß, wo was immer ist und dass man sich einfach so auskennt mit seinem eigenen Handy. (weiblich, 16 Jahre, Expeditive)

Also es ist halt schon peinlich, wenn man nicht weiß, was Google ist, aber das weiß ja sowieso jeder. Was man wissen muss, dass es so was wie YouTube gibt, so eine Internetplattform, wo man YouTube-Videos gucken kann. Und halt soziale Medien auf dem Rechner, dass es das gibt. Aber jetzt zum Beispiel Computersprache mit Nullen und Einsen, das muss man nicht wissen. (männlich, 14 Jahre, Materialistische Hedonisten)

Sich im Internet sicher bewegen und selbst schützen zu können ist für Jugendliche von hoher Bedeutung. Hier zeigen sie auch den größten Bedarf nach mehr Information und Unterstützung. Es geht ihnen nur bedingt um den rein technischen Schutz, bei dem sie auf gängige Software vertrauen. Ihnen ist klar, dass es vor allem darauf ankommt, wo und wie man sich im Netz bewegt und welche Angebote man nutzt. 
Dass Daten im Internet "nie wieder gelöscht werden können“ ist ihnen bewusst. Sie betonen zwar, dass man daher aufpassen muss, verfügen aber nicht über klare Kriterien, was man im Internet macht oder lieber sein lässt. Es werden kaum Seiten oder Dienste genannt, die konkret zu vermeiden sind, vielmehr wird auf persönliche Erfahrungswerte aus dem Bekanntenkreis rekurriert. Insgesamt überwiegt eine pragmatische Sorglosigkeit, dass einem selbst nicht viel passieren kann.

(1) Dass man aufpasst, dass man nicht auf Seiten kommt, wo man sich Viren oder Trojaner einfangen könnte, dass man halt einfach ein ordentliches Schutzprogramm hat, was einen dann auch warnt. Dann kann einem eigentlich nicht so viel passieren. (weiblich, 16 Jahre, Experimentalistische Hedonisten)

( I ch kenne Freunde, die sich durch Streaming Viren auf ihren Computer geholt haben. Das sollte man bedenken. Aber viel muss man da eigentlich nicht wissen. (weiblich, 17 Jahre, Adaptiv-Pragmatische)

(7um Beispiel so Cybermobbing und so, das sollte man halt alles wissen und nicht einfach dumm reinschauen. (männlich. 16 Jahre, Materialistische Hedonisten)

Großes Augenmerk richten Jugendliche auf die Frage, welche persönlichen Daten sie in welcher Form im Internet preisgeben. Es herrscht Einigkeit, dass man nicht alles wahllos postet - vor allem nicht, wenn man unsicher ist, wer der Empfänger ist. Mit der Veröffentlichung privater Daten ist man laut eigenem Bekunden daher vorsichtig, auch wenn sich dies zumeist ausschließlich auf Fotos und persönliche Informationen (z. B. Beziehungsstatus) bezieht. Die Angst vor der Veröffentlichung peinlicher Details ist groß, daher schätzen Jugendliche Informationen über ihr Privatleben (wann, wo, was und - vor allem - mit wem) wertvoller ein als soziodemographische Daten.

(7) Dass man in Facebook nicht alles posten sollte. Man sollte jetzt zum Beispiel, wie in meiner Situation: "Yeah, ich bin allein zu Hause heute Nacht. Yippie, Party kann steigen." Sowas sollte man nicht unbedingt 
auf Facebook posten. Weil das lädt dann Einbrecher ein. (weiblich, 17 Jahre, Konservativ-Bürgerliche)

( Im Internet viele Sachen, also so etwas wie Datenveröffentlichung. Darum mache ich das eigentlich auch nie. (männlich, 15 Jahre, Materialistische Hedonisten)

( Ja, ich finde, man sollte halt auf jeden Fall achtsam damit umgehen, weil halt gerade im Internet oder sozialen Netzwerken, ist halt schon so, dass man da aufpassen muss, was man hochlädt. Und auch zum Beispiel jetzt bei Handys, finde ich auch wichtig, dass man sich überlegt, zum Beispiel, dass man jetzt den Facebook Messenger nicht runterlädt, weil der überwacht einen ja dann und kann auf alles zugreifen und auf sowas, finde ich, sollte man halt schon achten. (weiblich, 17 Jahre, Adaptiv-Pragmatische)

Ich glaube, dass man nicht zu viel von sich preisgibt. Also dass man nicht zu viel reinstellt. Und vor allem keine Sachen, die niemand von einem erfahren sollte wie zum Beispiel den Wohnort. (weiblich, 14 Jahre, Adaptiv-Pragmatische)

Auszüge aus Gesprächen zwischen Jugendlichen zum Thema Digitale Medien
Interviewerin: weiblich, 16Jahre, Adaptiv-Pragmatische

GIBST DU VON DIR VIEL IM INTERNET PREIS? WAS HAST DU ZUM BEISPIEL AUF FACEBOOK ANGEGEBEN?

(7) Also auf Facebook hab ich mein Profil, also meinen Namen, dann hab ich ein Bild, dann hab ich über 600, 700 Fotos drin, Handy-Uploads. Und dann steht da natürlich, wann ich geboren bin, wo ich wohne, also direkt nicht welche Straße, aber in welchem Bezirk ich wohne. Ob ich verheiratet bin oder ob ich verlobt bin, das steht nicht, ob ich in fester Beziehung bin, das steht auch nicht. Ansonsten poste ich natürlich, egal wo ich bin, hier bin ich, da bin ich, egal ob ich bei [NAME EINER BEKLEIDUNGSMARKe] bin oder bei [NAME eINES KaUfHAUSES] bin, poste ich das. Es ist vielleicht nicht gut, dass ich alles poste und dass ich alles freigebe, aber dafür ist das ja da, und ich find das gut. 
ABER FINDEST DU'S NICHT GEFÄHRLICH? ALSO WENN MAN EIN FOTO HOCHLÄDT, WIRD'S JA NIE AUS DEM INTERNET GELÖSCHT, UND DAS IST JA AUCH EIN BISSCHEN RISKANT.

( Ja, natürlich ist es riskant. Aber heute schicken wir über WhatsApp auch Sachen. Hast du mal die WhatsApp-AGBs gelesen? Da wird auch irgendwo auf einer externen Festplatte bestimmt alles gespeichert, und dann sollte man auch keine Fotos schicken. Natürlich ist das riskant, aber so lang ich keine Nacktfotos von mir gebe, ist es mir egal.

\section{Interviewer: weiblich, 17 Jahre, Expeditive}

WAS POSTEST DU SO, UND WO?

( Also auf Facebook poste ich eigentlich gar nichts, höchstens Instagram, Bilder, aber sonst eigentlich gar nichts. Sonst habe ich keine anderen sozialen Netzwerke.

\section{GIBT'S WAS, WAS DU NICHT POSTEN WÜRDEST?}

(J) zu private Dinge, oder meinen ganzen Tagesablauf, was ich mache, wo ich bin. Also man kann ja z. B. Orte markieren, entweder bei Instagram oder Facebook, würde ich nicht machen, weil es sieht jeder und es muss nicht jeder wissen, wo man sich gerade aufhält. Ja, oder vielleicht auch Bilder oder irgendwelche Sachen, wo andere Leute mit drauf sind oder beteiligt sind, wenn man die nicht vorher gefragt hat.

WARUM?

( Ja, ich finde, das ist zu privat. Oder auch so, Telefonnummern, Emails würde ich auch nie öffentlich machen, weil einfach die Leute, mit denen man Kontakt hat und für die es wichtig ist, dass die Leute deine Daten haben, die wissen die Daten oder denen kann man persönlich es auch erzählen, wenn man sie sieht. 


\section{Die Beherrschung von Programmen und Programmiersprachen gilt als Spezialkenntnis, die persönlich kaum relevant ist.}

Den Umgang mit Software bzw. komplexeren Anwendungen (MS Office, Grafikprogramme) sehen Jugendliche nicht als besonders relevante Kenntnis im Bereich der Medienkompetenz an. Diese Fähigkeiten gehören für sie nicht zum Repertoire der Aktivitäten, die sie sich selbst (freiwillig) beibringen. Diese Kompetenzen werden überwiegend in der Schule (teilweise unterstützt durch Eltern) vermittelt und stoßen auf mehr oder weniger Begeisterung. Jugendliche mit höherem (angestrebtem) Bildungsabschluss sehen hier am ehesten eine zumindest künftige Relevanz dieser Kenntnisse.

Insbesondere Programmieren gilt als besonders komplex und findet wenn überhaupt - fast ausschließlich in der Schule statt. Bis auf wenige Ausnahmen (fast ausschließlich handelt es sich dabei um Jungen) haben sich die Befragten kaum damit auseinandergesetzt, weil die Komplexitätsbefürchtung hoch und die Frustrationsgrenze niedrig liegt.

Typische

Zitate zur Illustration

Also hatte ich auch mal so einen Informatikkurs und auch in der Oberstufe habe ich jetzt ein Jahr Informatik gemacht und weiß halt, was man wirklich jetzt alles machen kann am Computer, zum Beispiel mit irgendwelchem Programmieren, was aber schon ziemlich schwer ist, muss ich zugeben, aber schon interessant. (männlich, 17 Jahre, Experimentalistische Hedonisten)

( Oder jetzt mit der Schule auch mit diesem Microsoft Office, in Mathe haben wir da noch ein Programm bekommen, wo wir eine Tabelle aufbauen müssen. Wir müssen eine Tabelle aufbauen, in einem Programm, das ich überhaupt nicht kenne. Da muss ich wirklich daran arbeiten. Aber das mache ich in der Schule. (weiblich, 14 Jahre, Adaptiv-Pragmatische)

( Ja, es war interessant, aber ich glaube Programmieren ist für mich persönlich zu trocken, als dass ich es weiter machen würde. (...) Ja, es ist anstrengend, an sich auch die ganze Zeit. Man muss halt immer die Sprache des Computers beherrschen, die Befehle so eintippen, macht 
halt nichts anderes, als einfach nur Befehle eintippen und dann hoffen, dass es dann läuft. (männlich, 16 Jahre, Adaptiv-Pragmatische)

\subsection{Lernen mit digitalen Medien in der Schule}

\section{Lernen mit digitalen Medien macht mehr Spaß als Paper-Pencil-Unterricht.}

Dass das Lernen mit digitalen Medien Vorteile bietet, ist Konsens bei den Jugendlichen. Sie sehen vor allem die Vielfalt der Gestaltungsmöglichkeiten, die automatischen Korrekturfunktionen und den Wegfall des mühsamen händischen Schreibens als unschlagbaren Vorteil. Insbesondere für Jugendliche aus Elternhäusern, in denen digitale Medien kaum oder wenn, dann nur als Entertainmentzentrale eine Rolle spielen, sind diese besondere Attraktoren, die durch ihre reine Verfügbarkeit ein erhebliches Maß an Aufmerksamkeit auf sich ziehen.

( Man kann Hausaufgaben auf dem Computer machen mit Word und halt Präsentationen mal, was ich persönlich auch viel praktischer finde, weil es mit der Rechtschreibung immer eine Kontrolle gibt, aber es geht im Endeffekt auch von der Schrift her, weil man halt eben nochmal schnell im Internet gucken und ein Bild dazu machen kann. Man kann mal eben schnell alles unterstreichen. Und es sieht nicht aus, wie kurz mal alles übergestrichen, wenn man mal etwas falsch gemacht hat. (weiblich, 17 Jahre, Adaptiv-Pragmatische)

(1) Es geht schneller und ich kann mich zum Beispiel viel besser konzentrieren, wenn ich das auf dem Computer schreiben soll. Das ist einfach. Da kann ich mir das besser merken. Und man kann es vor allen Dingen hinterher lesen. (weiblich, 17 Jahre, Konservativ-Bürgerliche)

Wir sind einfach auf eine Seite gegangen. Da haben wir die Ländernamen alle durchgemacht in Europa und mussten dann die Hauptstädte lernen und die Ländernamen. Dann bestimmte Flüsse, Berge, dann 
Königreiche. Und irgendwann haben wir angefangen mit Afrika. Das ging immer so weiter. Das hat mir Spaß gemacht. Also solche Sachen finde ich schon schön, weil man lernt auch dabei. Das klingt kindisch, weil spielend lernen sozusagen in dem Sinne. (weiblich, 16 Jahre, Konservativ-Bürgerliche)

[INT: UND WÜRDEST DU DIR WÜNSCHEN, DASS DAS IN DER SCHULE MEHR BENUTZT WIRD?] Ja, schon. Macht mehr Spaß und ist einfacher. (weiblich, 14 Jahre, Materialistische Hedonisten)

\section{Schulen sind aus Sicht der Jugendlichen defensive Akteure in punkto digitales Lernen.}

Spontane Assoziationen zum Thema "Digitale Medien in der Schule" drehen sich vor allem darum, welche Geräte in der Schule wo und wann verboten sind und was im Internet aus Sicht der Lehrer alles gefährlich ist. Ein Tag ohne Smartphone ist für die Jugendlichen schwer zu ertragen. Dennoch akzeptieren sie, dass private digitale Geräte während des Unterrichts - eigentlich - verboten sind. Man hält sich mehr oder weniger an diese Abmachungen. Mit zunehmendem Alter der Schüler werden die Reglementierungen seitens der Lehrer weniger stark eingefordert.

Typische Zitate zur Illustration
Also bei uns war es so, wer sein Smartphone rausgepackt hatte im Unterricht, da gab es eine erste Verwarnung. Bei der zweiten wurde es halt eingesammelt und nach der Stunde wiedergegeben. Sonst haben die im Unterricht halt nur rumgespielt. (männlich, 16 Jahre, Materialistische Hedonisten)

( Nee, es kommt natürlich auf die Lehrer an, aber man darf die Handys eigentlich nicht haben. Man muss schauen, dass der Lehrer es nicht sieht. Musik hören darf man natürlich auch nicht, weiß nicht. Sobald Schule aus ist, kann man das natürlich alles machen, aber während der Schulzeit nicht. (weiblich, 15 Jahre, Experimentalistische Hedonisten) 
(1) Früher haben die immer was gesagt, und das machen die bei den kleinen Kindern, glaube ich, immer noch. Und wenn es im Unterricht zum Beispiel klingelt oder man spielt heimlich und es geht der Ton an, dann wird es, glaube ich, auch noch eingezogen und im Sekretariat darfst du es abholen. (männlich, 14 Jahre, Materialistische Hedonisten)

Zwar wird im Unterricht mit digitalen Medien gearbeitet, allerdings nur phasenweise und fächerspezifisch. "Computer" ist zumeist ein Extra-Fach, zum Beispiel in Form spezifischer Kurse oder Wahlpflichtfächer. Der Umgang mit digitalen Medien wird nicht fächerunabhängig selbstverständlich integriert oder thematisiert. Überwiegend ist der Einsatz nutzenorientiert und anlassbezogen, insbesondere für die Vorbereitung von Referaten, das Schreiben von Bewerbungen o. Ä.. Mit Blick auf die Aktivitäten dominiert aus Sicht der Jugendlichen der Fokus auf das korrekte Bedienen von Bürosoftware. Digitale Medien finden somit zu definierten Zeiten und in bestimmten Kontexten Anwendung, und zwar für Lernziele, die nicht primär auf den Erwerb von Kompetenzen im Umgang mit Medien ausgerichtet sind. Medien dienen als Mittel zum Zweck, häufig auch zur Belohnung, Entspannung oder zur generellen Motivation.

( Im täglichen Unterricht eigentlich gar nicht. Nur wenn wir vielleicht eine Präsentation machen können oder machen müssen. Wenn wir es am Computer machen und präsentieren wollen, dann haben wir die Möglichkeit, einen USB-Stick mitzubringen und die Information herunterzuladen. (männlich, 14 Jahre, Adaptiv-Pragmatische)

(7) Es gab immer schon so Computertechnik in der Realschule. Da haben wir dann eigentlich eher an Word gearbeitet, wie das alles geht. (männlich, 17 Jahre, Adaptiv-Pragmatische)

(7) Das finde ich ein bisschen schade, wir haben nur einmal in der Woche Informatik. Das sind solche Sachen am Rechner. Ich finde, so was sollte man wirklich ein bisschen öfter machen. Zwei Stunden die Woche wären cool. Vor allen Dingen, wenn man so was jetzt nicht versteht. Ich vergesse das auch immer so schnell, jetzt zum Beispiel: Da oben geht man auf Speichern, diese und diese Maustaste ist ein 
Kurzbefehl Drucken. Also so was. (männlich, 14 Jahre, Materialistische Hedonisten)

(>) Yeah, heute gehen wir mal in den Computerraum, das ist etwas ganz Besonderes. (weiblich, 15 Jahre, Adaptiv-Pragmatische)

Auszüge aus Gesprächen

zwischen Jugendlichen zum Thema Lernen mit digitalen Medien

\section{Interviewerin: weiblich, 17 Jahre, Expeditive}

DENKST DU ÜBERHAUPT, DASS DIE LEHRER KOMPETENT GENUG SIND, DIGITALE Medien ZU NUtZEN? KenNEN DIE SICH DAMIT GUT GENUG AUS?

( Das ist schwierig. Ich denke, jüngere Lehrer, Referendare, die vielleicht gerade fertig sind mit ihrem Studium, eher als ein Lehrer, der jetzt schon 30 oder 40 Jahre in seinem Job arbeitet, weil derjenige ja mit dem Internet und mit den ganzen modernen Medien nicht so aufgewachsen ist. Und ich denke, wenn man jetzt in den Schulen alles umstellen würde, dass es nur noch iPads gibt, wo man darüber seine Klassenarbeit schreibt oder so, kann ich mir schon vorstellen, dass ältere Lehrer damit vielleicht erst mal überfordert sind.

\section{Interviewer: weiblich, 17 Jahre, Sozialökologische}

INWIEFERN SIND DIGITALE MEDIEN SCHON IN DEINEN LERNPROZESS INTEGRIERT? Also ZUM Beispiel für HAUSAUfgaben, ZUM KLAUSUREN VORBEREITEN, ODER ZUR NACHBEREITUNG VOM UNTERRICHT.

( Also auf jeden Fall sind sie sehr doll integriert, weil ich Hausaufgaben eigentlich grundsätzlich mit dem Internet mache oder am PC, weil ich meine Hausaufgaben meistens am PC niederschreibe. Und wenn man natürlich irgendwelche Informationen zu einem Thema braucht, dann fragt man das Internet. Und ansonsten zu Vorträgen wird auch alles digital gemacht, Plakate sind bei mir eigentlich ausgestorben. Und Klausurvorbereitung, wenn man sich jetzt eine Gliederung macht, bevor man anfängt zu schreiben. Solche Informationen zieht man dann eigentlich auch aus dem Internet. Oder Kontextwissen.

\section{Wie ARbeitet denn deine eigene Schule mit digitalen Medien?}

( Also wir haben jetzt ganz viele Smartboards bekommen, aber das ist irgendwie so als hätten wir keine bekommen, weil die kann sowieso 
keiner benutzen. Und kein Lehrer versteht die, weil es einfach keine Einweisung gab, also keine richtige Schulung. Und jetzt stehen die Smartboards da und sind nur zum Angucken da. Unser Computerraum, die $P C$, die sind ganz schön alt. Und ansonsten arbeiten wir immer noch mit diesen superalten verstaubten $\mathrm{OH}$-Projektoren, wo jeder dritte nicht funktioniert und wo jeder zweite ein komisches Bild projiziert. Also meine Schule ist da nicht wirklich sehr hinterher.

\section{Ob digitale Medien in der Schule eine zentrale Rolle spielen sollten, ist eine Frage der Lebenswelt.}

Eine stärkere Einbindung digitaler Medien in den Unterrichtsalltag wünschen sich vor allem Jugendliche aus den hedonistischen und prekären Lebenswelten. Man erhofft sich einen höheren Unterhaltungswert, sowohl im Unterricht, als auch in den Pausen. Gerade dort, wo Jugendliche von Haus aus weniger gut mit digitalen Geräten jenseits von Spielekonsolen und Smartphones ausgestattet sind, weil die berufliche Sphäre der Eltern kaum digital geprägt ist, ist der Einsatz von Medien im Unterricht nahezu die einzige Möglichkeit, überhaupt mit Rechnern und Laptops in Kontakt kommen zu können. Was dann genau mit diesen Geräten im Unterricht inhaltlich passiert, ist weniger relevant, von primärem Interesse ist die reine Verfügbarkeit. Eine große Faszination üben hier prominente IT-Marken aus, die mit urbanem Lifestyle und Coolness assoziiert sind und auch im Freundeskreis anerkennend registriert werden.

( Ich finde, man sollte schon mit Duden Wörter suchen können, aber wenn man es kann, kann man auch einfach mal im Handy nachgucken, weil es einfach zeitsparend ist. Wenn man jedes Wort nachschlagen muss, wenn man es absolut nicht kann, das ist einfach nervig. (weiblich, 16 Jahre, Experimentalistische Hedonisten)

( Es wäre natürlich endgeil, wenn man einen Laptop hätte, weil den kann man auch mit nach Hause nehmen. Oder man kann Musik hören im Unterricht. Wer meint, das machen zu müssen, dann den ganzen Stoff verpasst, kann er ruhig machen. Ist ja Lehrersache. Aber so wäre es 
schon cool, wenn es einem nicht direkt abgenommen würde. Oder in den Pausen, dass man schon Musik hören kann, das wäre schon cool. (GP 24, weiblich, 15 Jahre, HS, Experimentalistische Hedonisten)

( J) Ja, also bei uns war es so, dass wir in Deutsch und in Ethik sehr viele Stunden hatten, wo wir ein Thema bekommen haben. Zum Beispiel in Deutsch hatten wir eines. Man sollte eine Kommunikation darstellen. Und da haben wir so Videos aufgenommen. Da habe ich das dann zusammengeschnitten usw. Das haben wir dann präsentiert. Und in Ethik habe ich einen Werbespot gemacht für Zivilcourage. Das macht voll Spaß. (männlich, 16 Jahre, Materialistische Hedonisten)

( Also es gibt ja in manchen Schulen in Berlin eine Computerklasse. Da benutzt man ein MacBook. Und ich würde es also gut finden, wenn man mit einem Computer oder halt einem Laptop oder MacBook dann da arbeitet. Da ist das ein anderer Effekt im Unterricht. Da hat man einfach ein besseres Gefühl. Das ist jetzt einfach was Neues, so. Man denkt: Wow, voll schön! (männlich, 16 Jahre, Materialistische Hedonisten)

Also ich finde es gut. Vor allem wenn man halt wirklich was nachgucken kann, wenn man halt jetzt noch mal ein Thema hat. Zum Beispiel im Geschichtsunterricht war es letztens mal, mir ist halt so eine Frage aufgekommen, halt so warum die, Freßgass' jetzt, Freßgass' heißen, da hat man halt kurz mal nachgeguckt und konnte man dann auch gut mitreden danach. Also habe ich mich kurz informiert. Ja, das ist auf jeden Fall cool, ja. (männlich, 17 Jahre, Experimentalistische Hedonisten)

Jugendliche aus postmodernen Lebenswelten hingegen wünschen sich nicht unbedingt mehr digitale Medien in der Schule - teilweise ist es ihnen egal, teilweise grenzen sie sich davon ab. Als Begründung werden dabei Aspekte wie fehlende Effizienz, da "eh jeder nur auf YouTube und so weiter" geht, sowie der Abbau der eigenständigen Denkkompetenz genannt. Man brauche nicht noch mehr Computer in der Schule, zwar biete das Arbeiten damit gewisse Vorteile, andererseits seien diese nicht besonders groß.

Außerdem wird kritisiert, dass bestimmte "analoge Skills" (wie z. B. das Recherchieren in Büchern) verloren gehen könnten und dass Internet 
die Menschen immer passiver ("fauler") und „immer dümmer macht, weil man immer weniger selbst nachdenken muss". Dabei grenzen sie sich vereinzelt auch von denjenigen Jugendlichen ab, die sich von digitalen Möglichkeiten weitestgehend "abhängig" gemacht haben und ohne GPS-Funktion nicht mehr den Weg nach Hause finden würden. Jugendliche in postmodernen Lebenswelten sind von Haus aus technisch gut ausgestattet und ihre Eltern pflegen einen selbstverständlichen Umgang mit digitalen Medien. Online und vernetzt zu sein ist für Eltern in diesen Lebenswelten Normalität - häufig schlicht auch berufliche Notwendigkeit. Für ihre Kinder sind Medien daher nicht per se Unterhaltungs-Tools, sondern auch Arbeitsinstrumente. Ferner sind diese Jugendlichen weitgehend gesättigt und die bloße Präsenz eines Smartboards löst bei ihnen noch keine Begeisterungsstürme aus. Da der Einsatz von Medien allein für sie noch keinen Zweck erfüllt, hinterfragen diese Jugendlichen den Nutzen des konkreten Einsatzes. Was daran macht den Unterricht besser?

(8) Früher hat man im Lexikon nachgeschaut, und ich glaube, ganz viele wissen nicht mehr, dass es das auch gibt oder können damit nicht umgehen. Deswegen bleibt das immer wichtig in Zukunft. (weiblich,

Typische Zitate zur |llustration 16 Jahre, Expeditive)

( Ich muss sagen, ich vermisse das Internet in der Schule nicht. Jeder Lehrer macht es eh anders. Man könnte vielleicht ein bisschen mehr mit Computern arbeiten. Aber wenn man dann im Computerraum ist, macht sowieso nicht jeder das, was er am Computer machen soll. Deswegen kann ich es auch verstehen, dass die Lehrer nicht so oft die Computer nutzen. (weiblich, 17 Jahre, Adaptiv-Pragmatische)

( In USA oder Australien haben die Schüler ihren eigenen Laptop immer und darüber werden alle Hausaufgaben geschickt und sie können damit auch mit dem Lehrer kommunizieren. Das ist vielleicht manchmal ganz hilfreich, gerade was so die Hausaufgaben angeht und so. Aber sonst ist es, glaube ich, auch nicht wichtig. Das braucht man eigentlich nicht, extra seinen Computer nur für seine Schule zu haben. (weiblich, 16 Jahre, Expeditive) 
Ich würde es wirklich so lassen, dass man die Medien auch nicht komplett mit einbringt, sondern dass dies Schreiben nicht komplett durch das Eintippen ersetzt wird. Dass man, wie gesagt, zu Präsentationen oder wenn man was nachgucken will, Medien dann nimmt, aber sonst, dass wirklich nicht jeder irgendwie seinen eigenen Laptop dann mitbringt und dann irgendwie die ganze Zeit vor dem Ding sitzt in der Schule. (männlich, 17 Jahre, Experimentalistische Hedonisten)

( Ich merke, das wird jetzt alles viel mehr so, dass man mit Tablets anfängt und ich finde das eigentlich echt ein bisschen viel. Also ich würde mir wünschen, dass es so bleibt, dass man auf dem Block ganz normal schreibt. (männlich, 16 Jahre, Adaptiv-Pragmatische)

( Ich finde, am Gymnasium muss das nicht sein. Da fehlt vielen Schülern die Motivation, würde mir auch fehlen, wenn alle Laptops haben, würde die Hälfte auf YouTube gehen, sich Instagram angucken, ich würde Schuhe angucken oder wie Köln spielt, welche Aufstellung, wer verletzt ist, welche Autos jetzt neu rauskommen. Da fehlt die Motivation. (männlich, 15 Jahre, Materialistische Hedonisten)

(3) Also wir haben in der Schule jetzt so Whiteboards bekommen, so riesengroße Bildschirme mit Touchscreen und sowas, finde ich ganz in Ordnung, aber auch irgendwie unnötig. Also ich finde eine Tafel genauso gut. (weiblich, 17 Jahre, Experimentalistische Hedonisten)

( Man wird dümmer dadurch, finde ich. Also durch das Internet wird man ja dümmer. [INT: WIESO DAS, ERKLÄR MAL?] Zum Beispiel wenn ich etwas wissen möchte, google ich es direkt und denke gar nicht selbst darüber nach. Ich finde es eigentlich schade, aber es hilft mir. (männlich, 16 Jahre, Adaptiv-Pragmatische)

\section{Die technische Ausstattung der Schulen kritisieren Jugendliche als nicht ausreichend und veraltet.}

Jenseits der unterschiedlichen Präferenzen, ob und wieviel Einsatz von Medien in der Schule sinnvoll und wünschenswert ist, sind sich Jugendliche aller Lebenswelten in einer Sache einig: Wenn sie eingesetzt werden, 
sollten sie auf dem aktuellen Stand der Technik sein. Veraltete Geräte und entsprechende Software gelten als "nutzlos".

Smartboards und Dokumentenkameras werden inzwischen als "normale" Ausstattung eingeordnet und haben sich als Tafelersatz etabliert. Darüber hinaus sind Jugendliche zuhause jedoch in technischer Hinsicht meist besser ausgestattet als in der Schule. Die schulischen Computerräume werden zudem als chronisch überlastet und zu reglementiert wahrgenommen.

Es wäre wichtig, dass die Technik auf dem neuesten Stand ist. Aber das ist schwierig. Man kann nicht so schnell neues Zeug für so viele Schüler kaufen, damit die Technik der Schule gleichgesetzt mit der priTypische Zitate zur |llustration vaten Technik ist. Das wäre aber schon wichtig. (weiblich, 15 Jahre, Expeditive)

( Also jetzt hat ja glaube ich fast jede Schule so ein Smartboard. Also das ist keine Tafel mehr so, das ist wirklich so wo man antippen kann alles. Und da kann man wirklich alles machen damit. (männlich, 16 Jahre, Adaptiv-Pragmatische)

(7) Wir haben Medienräume in der Schule, also Computerräume mit den neuesten Sachen. Naja, für uns Schüler sind das nicht wirklich die neuesten Sachen, aber sie halten sich wirklich ganz gut. In meiner alten Schule waren solche Boards aufgestellt, wo man anstatt mit Kreide mit Stiften schreibt. Meine alte Schule hatte die schon gefühlt seit drei Jahren. (weiblich, 16 Jahre, Experimentalistische Hedonisten)

(7) Aber in meiner damaligen Schule war es so, wenn man Präsentationen hatte, durfte man seinen Laptop mitbringen und da was machen oder man hatte seinen USB-Stick dabei und hat es an den Beamer angeschlossen. (männlich, 17 Jahre, Experimentalistische Hedonisten) 


\section{Seitens der Lehrer werden digitale Kompetenzen teilweise vorausgesetzt.}

Beim Einsatz von digitalen Medien in der Schule erwähnen Jugendliche, dass die Lehrer zumeist davon ausgehen, dass bestimmte Geräte zu Hause vorhanden sind und bei Bedarf in den Unterricht mitgebracht werden können (z. B. Laptops). Auch dass man weiß, wie mit diesen Geräten umzugehen ist, wird häufig implizit vorausgesetzt. Die digitale Ausstattung und das digitale Kapital des Elternhauses werden somit als Ressource in die Schule eingebracht, ohne dass dabei klar ist, welche Fähigkeiten von den Jugendlichen genau erwartet werden.

(7) Also dann was der Lehrer halt immer sagt, wenn er zum Beispiel sagt, wir sollen jetzt im Internet recherchieren über zum Beispiel aktuell das Thema Flüchtlinge oder über das Mädchen, was jetzt in Meißen verschwunden ist. Das ist halt immer recherchieren. Dann tun wir das dann immer irgendwie kopieren und zeigen es dann immer dem Lehrer. (männlich, 14 Jahre, Konservativ-Bürgerliche)

( Handys dürfen wir ab und zu benutzen, es kommt immer drauf an. Zum Beispiel auch wenn wir manchmal Kunst haben und ich eine Rose zeichnen möchte, dann frage ich den Lehrer, ob ich kurz mit dem Handy eine Rose googeln kann und sie abzeichnen kann. (weiblich, 14 Jahre, Experimentalistische Hedonisten)

\section{Die Jugendlichen sehen Schule - potenziell - als relevanten Akteur für Medienkompetenz bei Sicherheitsfragen.}

Dass man sich digitalen Medien in der Schule vor allem aus der Perspektive von Risiken und Gefahren nähert, wird von den Jugendlichen einerseits bedauert; auch die generelle Kritik an zentralen Anbietern und Plattformen ("die sagen immer nur "Facebook ist böse' und so") erzeugt eine ablehnende Haltung. Andererseits sind Risiken im digitalen Raum als Thema von hoher persönlicher Relevanz und Jugendliche möchten mehr darüber erfahren. In der Schule lernt man aus Erfahrung der Jugendlichen 
insbesondere, wie man sich vor jugendgefährdenden Inhalten und dem Abgriff persönlicher Daten schützen kann oder wohin man sich bei Mobbing wenden kann. Dies geschieht teilweise auch durch den Unterrichtsbesuch von externen Experten (z. B. Polizisten, Live-Hacker).

(7) Also wir hatten das früher voll viel in der Schule. Da hatten wir auch immer so Berater oder sowas, die einen so aufklären. Es war auch mal ein Polizist da, und auch die Lehrer reden viel mit uns, und irgendwann Typische Zitate zur Illustration kennt man es dann halt, weil die immer das gleiche sagen, die Eltern natürlich auch. (weiblich, 17 Jahre, Adaptiv-Pragmatische)

( Auf dem Gymnasium hatten wir oft das Thema Internet. Da wurden wir auch richtig oft schon belehrt sozusagen. Da war auch einmal die Polizei da, hat das erklärt und hat Filme gezeigt. (weiblich, 16 Jahre, Experimentalistische Hedonisten)

( Da war so ein Projekttag über Medien. Das waren zwei Tage, wo wir uns darüber unterhalten haben, auch mit Fachleuten, die in unsere Schule kamen und uns das erklärt haben. Das war schon gut und das meiste wusste man dann, weil man das über Freunde und Eltern und so erfahren hat. Aber das noch mal von einem Fachmann zu erfahren, Cybermobbing ist ja auch gerade aktuell und da will man ja selber nicht reingeraten. (männlich. 15 Jahre, Materialistische Hedonisten)

( Also nur in der Realschule jetzt über die Sicherheit, aber jetzt nicht wirklich viel. (männlich, 16 Jahre, Adaptiv-Pragmatische)

\section{Jugendliche möchten digitale Medien nicht nur nutzen, sondern auch verstehen.}

Jugendliche wünschen sich einen weniger gefahrenzentrierten Unterricht, der die Chancen von digitalen Medien aufzeigt und konkrete Kriterien vermittelt, wie diese Chancen umgesetzt werden können, ohne sich allzu hohen Risiken auszusetzen. Jugendliche würden beispielsweise gerne lernen, wie man das Internet benutzt, worauf man achten muss und wie man sich schützt. Lehrer werden vorrangig als Mahner 
aufgefasst, die sagen, dass man aufpassen muss, jedoch nicht genauer erklären, nach welchen Maßstäben man dabei vorgehen soll. Hier wird auch ein deutliches Kompetenzdefizit wahrgenommen: Welchen Seiten im Internet man trauen und nicht trauen kann, wissen die Lehrer laut Jugendlichen selbst nicht.

Allgemein bemängelt wird, dass Technik zwar genutzt, jedoch nicht wirklich erklärt wird. Gern wüsste man, wie ein Computer oder das Internet eigentlich "funktioniert". Gewünscht wird ein stärkerer Fokus auf technische Aspekte (wie man beispielsweise Windows mit einem Apple-Handy verbindet), ein tieferes Verstehen der benutzten Programme und eine größere Relevanz der gelehrten Inhalte für das tägliche Leben.

Typische Zitate zur Illustration
Also bei Applegeräten, zum Beispiel, wenn man jetzt einen Apple Computer und ein Apple Handy hat, dann ist das schon ziemlich leicht. Also leichter als Windows-Gerät und Apple-Handy. Dass man zeigt, wie man sowas verbindet oder wie man es zusammen nutzt, oder wie man es überhaupt nutzt. Und nicht irgendwelche Apps zeigt, die besonders cool sind. (männlich, 17 Jahre, Adaptiv-Pragmatische)

(1) Wir arbeiten mit Computern, aber wir lernen nicht, wie man mit dem Computer umgeht. (männlich, 15 Jahre, Konservativ-Bürgerliche)

(7) Wir haben zwar auch Informatik, aber ich habe mir das alles relativ selber gut angeeignet, also welchen Seiten ich zum Beispiel vertrauen kann, dass die Informationen richtig sind. Und wie ich am besten nach etwas suche. Ich denke aber, das sollte man auch in den Unterricht mit eingliedern. Und es gibt auch viele Lehrer, die damit noch nicht umgehen können. (weiblich, 15 Jahre, Expeditive)

( Und dann vielleicht auch über ein paar Gefahren des Internets aufklären: Da und da könnte man einen Virus bekommen, bei solchen Angeboten müsst ihr aufpassen, das ist Fake! So etwas. (weiblich, 15 Jahre, Sozialökologische)

Videobearbeitung und vielleicht auch noch ein bisschen mehr im Info-Unterricht über Schutz im Internet. Darüber haben wir eigentlich relativ wenig gelernt, was man da für Programme nehmen kann oder vor 
welchen Seiten man vorsichtig sein muss, so etwas. [INT: HAST DU EINE IDEE, WIE DAS ABLAUFEN KÖNNTE, WENN MAN VIDEOSCHNITT UNTERRICHTEN WÜRDE?] Dass man einfach bei Windows Movie Maker erst mal Beispielbilder nimmt und die Grundlagen zeigt, dass die halt wissen, wie man so etwas macht und wie so etwas entsteht und dass sie dann ihre eigenen Videos schneiden können, vom Urlaub oder so. Das finde ich auch wichtig. (weiblich, 16 Jahre, Experimentalistische Hedonisten)

(1) Ich finde, man könnte einfach mehr Internet lernen, also wie man es benutzt und halt so, worauf man achten muss oder welche Möglichkeiten man hat, sich zu schützen. Also, weil die erzählen das einem immer, dass man aufpassen muss, aber letztendlich gezeigt wird es einem nie, wie man es macht. (weiblich, 17 Jahre, Adaptiv-Pragmatische)

\title{
4.5 Der Blick in die digitale Zukunft
}

\author{
Vor allem Jugendliche aus bildungsfernen Lebenswelten \\ glauben, dass das Internet für sie in der Zukunft eine weniger \\ wichtige Rolle spielen wird.
}

Online-Aktivitäten drehen sich bei Jugendlichen gegenwärtig hauptsächlich um Unterhaltung und Kommunikation. Das Internet ist Freizeitkosmos und Fenster zur Welt. Nur wenige Jugendliche assoziieren damit bereits den künftigen Berufsalltag. Auch die Schule ist offensichtlich kein Akteur, der aktuell entsprechende Vorstellungen konkretisieren oder Kompetenzen aufbauen hilft.

Dass das Internet vorwiegend unterhaltungsorientiert genutzt wird, ist aus Sicht der Jugendlichen bedingt durch ihre eigene Lebensphase - Unterhaltung und Kommunikation seien eben die wichtigsten Dinge im Leben der 14 bis 17-jährigen. Man geht davon aus, dass vor allem Games, stundenlanges Chatten, Durchscrollen von Feeds in den sozialen Netzwerken künftig immer mehr an persönlicher Bedeutung verlieren werden - "irgendwann kommt man halt aus dem Alter raus". Spätestens mit dem Übergang in das Berufsleben oder mit Gründung einer Familie rechnet man damit, weniger Zeit im Netz zu verbringen und 
Internetaktivitäten „runterzufahren“. Insbesondere YouTube und Facebook gelten als "Jugendangebote", die man als Erwachsener nicht mehr braucht. Dass man mit Freunden und Familie aber künftig weiterhin regelmäßig (auch) online in Kontakt ist (z. B. via Apps von Kurznachrichtendiensten wie WhatsApp) hält man hingegen für unverzichtbar.

Typische Zitate zur Illustration

(1) Ich denke, irgendwann kommt man aus dem Alter von Facebook, Instagram und Snapchat vielleicht ein bisschen heraus. Aber WhatsApp oder einzelne Nachrichtendienste behält man bestimmt. (weiblich, 17 Jahre, Adaptiv-Pragmatische)

(7) WhatsApp wird, wenn es das weiterhin gibt, immer eine Rolle spielen, weil ich damit mit meinen Freunden, mit meiner Familie und so in Verbindung stehe. Instagram vielleicht auch noch, um Bilder zu gucken. Auto Bild vielleicht auch. Aber Facebook nicht. (männlich, 15 Jahre, Materialistische Hedonisten)

(3) Es wird immer so sein, dass ich mit Freunden schreibe. Und das mit YouTube, das wird sich alles wieder legen, das wird irgendwann nicht mehr sein, auch das mit den Spielen. (männlich, 14 Jahre, Adaptiv-Pragmatische)

( Smartphone wird sicher bleiben, das wird sich nicht verändern. So Sachen wir PS4 wird bestimmt irgendwann immer unwichtiger. (männlich, 15 Jahre, Adaptiv-Pragmatische)

( Also ich denke mal, soziale Netzwerke wie Facebook werden bei mir überhaupt nicht mehr gebraucht. (männlich, 17 Jahre, Adaptiv-Pragmatische)

(7) Also das Internet auf jeden Fall, aber nicht mehr so Sachen, zum Beispiel YouTube oder Filme anschauen oder die ganze Zeit chatten, so was. (männlich, 15 Jahre, Materialistische Hedonisten)

Mit Blick auf das Zeitbudget gehen Jugendlichen somit davon aus, dass ihre Internetnutzung in der zeitlichen Intensität künftig eher abnehmen wird. Die Möglichkeit, das Internet dann lediglich anders zu nutzen (z.B. andere Netzwerke, andere Videoportale, neue Tätigkeiten, wie z. B. Einkäufe), wird kaum in Betracht gezogen. Wenn die Internetnutzung 
überhaupt als gleichbleibend oder zunehmend im eigenen Alltag eingeschätzt wird, dann mit Blick auf berufliche Notwendigkeiten. Dies hängt aber wiederum stark von der Lebenswelt ab. Im postmodernen Segment gilt es als selbstverständlich, dass der Berufsalltag online stattfindet und Erwachsene notwendigerweise Dinge "im Internet zu erledigen haben".

In bürgerlichen und prekären Lebenswelten geht man eher davon aus, dass das Internet im Berufsalltag kaum vorkommt und Online-Aktivitäten lediglich der freien Zeit vorbehalten sind.

( Ich glaube das Internet wird eine sehr große Rolle spielen. Vielleicht werde ich sogar damit arbeiten, gerade so als Mediendesignerin habe ich ja viel damit zu tun. (weiblich, 17 Jahre, Adaptiv-Pragmatische)

Typische Zitate zur Illustration

( Die werden auf alle Fälle noch eine sehr große Rolle spielen. Im Beruf aber auch in der Freizeit. In der Freizeit beschäftigt man sich ja viel damit. Und im Beruf wird das Hauptaugenmerk auch darauf liegen, damit man mit Kunden in Kontakt bleibt und auch bei Meetings. (männlich, 15 Jahre, Sozialökologische)

I Ich denke mal, ich werde sie einfach nicht mehr so viel benutzen, die Medien. Eigentlich einfach wahrscheinlich ab und zu noch das Internet oder für geschäftliche Sachen dann später irgendwann. Aber sonst das ganze Geschreibe und so wird abnehmen. (männlich, 16 Jahre, Adaptiv-Pragmatische)

(7) Internet und Smartphone sind nicht so wichtig in Zukunft. Also, weil später werde ich mich mehr für den Job interessieren, Frau, Kinder und so. (männlich, 14 Jahre, Prekäre)

( Man kommt in Zukunft nicht mehr am Internet vorbei. Wenn man das nicht drauf hat, ist man unten durch, egal ob privat oder beruflich. Da sollte man einen Kurs belegen, denn das braucht man wirklich. (weiblich, 16 Jahre, Experimentalistische Hedonisten)

$>\quad$ Wobei, es ist eigentlich jetzt nicht wirklich wichtig für die meisten in meinem Alter, aber es ist halt nur, weil sie es brauchen, weil sie ein bisschen süchtig danach sind, aber richtig brauchen tun es nur die Erwachsenen eigentlich. (männlich, 15 Jahre, Materialistische Hedonisten) 


\section{Jugendliche beklagen eine Entfremdung vom "real life" durch digitale Technologien - insbesondere bei Kindern.}

Bei aller Bedeutung, die man digitaler Teilhabe in der Zukunft beimisst, verlieren die Jugendlichen analoge Ressourcen nicht aus dem Blick. Es zeigt sich selbst bei Teenagern eine gewisse anti-digitale Sozialromantik bzw. eine "analoge Sehnsucht": Man vermisst den weniger digitalisierten Alltag „von früher". Teilweise fürchtet man, dass Kinder, die heute und in Zukunft aufwachsen, prägende "medienlose" Erlebnisse verpassen könnten, weil sie in einer digitalisierten Welt aufwachsen. Referenz bildet die aktuelle Ausstattung von kleinen Kindern mit digitalen Medien, die sie genau beobachten. Dass diese bereits mindestens so gut ausgestattet sind wie sie selbst, gefällt innen nicht teilweise aus Prinzip, teilweise aus Neid. Insbesondere Jugendliche aus Lebenswelten mit höherer Formalbildung weisen darauf hin, dass sie später bei den eigenen Kindern darauf achten werden, dass diese nicht zu früh mit digitalen Medien in Kontakt kommen.

Typische Zitate zur |llustration
Früher bei uns war das ja immer so: Wir haben im Buddelkasten gespielt, wir haben halt wie Kinder gelebt. Mit Barbies und Autos gespielt. Heutzutage haben die Kinder schon ein Smartphone, die noch in der ersten Klasse sind. Und ich hatte zum Beispiel mein erstes Handy in der siebten Klasse. Und dann haben die so viele Apps. Die werden in der letzten Zeit voll verwöhnt, die Kinder, die jetzt auf die Welt kommen. (weiblich, 14 Jahre, Materialistische Hedonisten)

( Ich sehe jetzt schon Fünftklässler mit einem iPhone herumlaufen. Und das ist nicht das iPhone 4 sondern das iPhone 6 oder 5. Das ist doch nicht mehr normal. Ich würde meinem Kind niemals in der fünften Klasse so ein Handy kaufen. Ich hatte damals eins, das war noch zum Klappen. Und das war auch nur für Notfälle, damit ich meine Eltern anrufen kann, wenn was ist. (weiblich, 16 Jahre, Materialistische Hedonisten)

(7) Also ich finde, wenn es sich so entwickelt, dann soll es sich so entwickeln. Ich habe jetzt kein Problem damit, aber fänd' es natürlich auch 
schön, wenn die kleinen Kinder wieder irgendwie Seilspringen würden und nicht nur am Handy sind, einfach, weil ich es so gewöhnt bin von früher. (weiblich, 17 Jahre, Experimentalistische Hedonisten)

( Ich weiß nicht, vielleicht hängen die Kinder dann so doll dran, dass sie irgendwie alle computersüchtig werden. (weiblich, 15 Jahre, Adaptiv-Pragmatische)

Schön, dass ihr ein besseres Handy habt als ich, das muss nicht sein und ich finde auch nicht, dass 5-Jährige mit einem iPad umgehen sollten, also wissen sollten wie man irgendwas öffnen kann, selbst wenn sie nur ihre Spiele spielen. Das finde ich komplett übertrieben und das würde ich auch nicht wollen, dass mein Kind das so früh lernt. (weiblich, 17 Jahre, Adaptiv-Pragmatische)

\section{Überwachung und Kontrolle als negative Folgen der Digitalisierung.}

Dass digitale Technologien gesellschaftlich als zentrale Infrastruktur immer wichtiger werden, sehen Jugendliche bei weitem nicht nur positiv. Sie sehen auch konkrete negative Entwicklungen der zunehmenden Digitalisierung, von denen sie glauben, dass sie ihr eigenes Leben - auch offline - in der Zukunft immer stärker bestimmen werden. Dass man Spuren im Internet hinterlässt und die eigenen Daten "für immer" im Netz verbleiben, nehmen Jugendliche nicht einfach nur zur Kenntnis. Sie fürchten wachsende Kontrolle durch diejenigen, die diese Daten "besitzen“. Jugendliche befinden sich dabei in einem Dilemma, denn auf der anderen Seite ist es für sie schlichte Notwendigkeit, Online-Dienste zu nutzen und damit im Netz Daten zu hinterlassen, um sozial nicht abgehängt zu sein. Zudem fühlen sie sich relativ sicher aus dem einfachen Grund, dass alle anderen auch ihre Daten im Netz haben.

Wenn es um den Schutz der eigenen Daten und die Privatsphäre geht, wünschen sie, dass der Staat die Unternehmen stärker kontrolliert und helfend eingreift, wenn Schaden entsteht. 
Eine weitere Facette ist der Verlust von Unabhängigkeit, insbesondere mit Blick auf das Berufsleben. Die Vorstellung, dass der Arbeitgeber durch moderne Kommunikationstechnologien auch in der Freizeit auf den Arbeitnehmer "zugreifen" kann, erfüllt Jugendliche mit Unbehagen - auch wenn dieser Aspekt eher vereinzelt genannt wird. Betont wird, dass es wichtig sei, dass man trotz digitaler Vernetzung noch „ein eigenes Leben" führen könne.

Typische

Zitate zur

Illustration

( Es wird auf jeden Fall mehr werden, also von den digitalen Medien her. Es wird immer größer, besser, schneller und überall wird sozusagen alles mit dem Internet vernetzt und man wird mehr und mehr ein gläserner Mensch, wie man so sagt. Das wird auf jeden Fall sich so verändern, aber das wird auch Initiativen geben, die halt dagegen ankämpfen werden. (männlich, 17 Jahre, Experimentalistische Hedonisten)

( Ich hoffe mal, dass es mehr Gesetze geben wird, die gerade Google oder so einschränken ein bisschen. Dass sie halt nicht alles überwachen können. Oder auch das mit der Werbung, dass man so angepasste Werbung bekommt. Wenn man das so sieht, ich habe zum Beispiel für meinen Abiball nach Kleidern gesucht im Internet und es kommen jetzt immer noch irgendwelche Vorschläge für Abschlussballkleider. Da fühlt man sich schon ein bisschen beobachtet. (weiblich, 17 Jahre, Expeditive)

( Dass man damit alles kontrollieren kann. Also jetzt Vorhänge auf und sowas, das gibt es ja schon. Aber ich glaube, das wird halt irgendwann viel zu viel sein, was man alles mit den Sachen machen kann. (männlich, 16 Jahre, Materialistische Hedonisten)

\section{Jugendliche haben nüchterne Visionen, wie die digitale Zukunft aussehen kann.}

Jugendliche machen sich keine konkreten Gedanken zur digitalen Zukunft. Zumindest fällt es ihnen schwer, spontan Ideen abseits von tradierten Science-Fiction-Vorstellungen zu entwickeln. Sie erwarten keine Überraschung, sondern gehen davon aus, dass alles „,irgendwie so weiter 
geht". Da ohnehin alle durchgehend online sind, kann man sich kaum eine Steigerungslogik ausmalen. Diese Erkenntnis mag zunächst überraschend sein, sie passt aber zu generell wahrgenommenen ersten Sättigungseffekten im Zuge beschleunigter Digitalisierungsprozesse und deckt sich mit Erkenntnissen der soziokulturellen Trendforschung des SINUS-Instituts.

Falls es doch eine digitale Überraschung geben sollte, haben die Jugendlichen kaum Ideen, worin diese bestehen könnte. Insbesondere scheint die Relevanz vieler möglicher neuer Produkte und Services im Alltag für die Jugendlichen fraglich. Vermutet wird hierbei eher, dass es lediglich darum geht, noch mehr Produkte verkaufen zu können, die doch niemand braucht. Vor allem in den Experimentalistischen und Hedonistischen Lebenswelten taucht teilweise Kritik am reinen Kommerz auf. In der Adaptiv-pragmatischen Lebenswelt verspricht man sich vor allen Dingen Alltagserleichterungen, d.h. Automatisierungs- und Beschleunigungsprozesse sowie mehr Convenience. Hier ist man auch am ehesten von einer generellen Fortschrittslogik überzeugt, z. B. dass ein Update bzw. eine neue Version eine Verbesserung der Vorgängervariante ist.

Das Internet der Dinge spielt in den Zukunftsvorstellungen der Jugendlichen nur vereinzelt eine Rolle; dominierend sind Weiterentwicklungen bestehender Geräte, Robotics und eingepflanzte Chips.

(7) Also es wird halt alles viel besser sein. Zum Beispiel WLAN, dass es dann vielleicht einen Router in der Stadt gibt, der dann an alle Haushalte liefert und du dann einfach was zahlst. Es wird halt alles verbessert, denke ich mal. Das sieht man ja jetzt schon. Wie zum Beispiel diese iPhone-Reihe von früher bis heute besser wurde. (männlich, 17 Jahre, Adaptiv-Pragmatische)

(1) Ich habe gar keine große Fantasie für so etwas. Aber ich glaube, da kann noch sehr viel kommen. (weiblich, 14 Jahre, Adaptiv-Pragmatische)

( Und es wird natürlich auch immer weitere Spielekonsolen geben, die sich immer weiterentwickeln. Jetzt mit CD und irgendwann sind in die Dinger selber Kameras eingebaut, die gibt es dazu jetzt. Und dann kann man darüber auch WhatsApp schreiben und die ganzen Apps 
runterladen. Es wird auf jeden Fall noch viel dazukommen. Es gibt bestimmt auch irgendwann so etwas, wo die das in die Haut einpflanzen. (männlich, 14 Jahre, Adaptiv-Pragmatische)

( Irgendwann muss man irgendwo nur dran langen und dann kommt irgendwas gebeamt her oder so, keine Ahnung. Richtig abgespaced halt. Naja, das macht mir schon Angst. (weiblich, 17 Jahre, Sozialökologische)

(7) Fliegende Autos auf jeden Fall. Die Handys werden immer größer. [INT: Aber fINDEST dU DAS AUCH REIZVoll?] Nein. Also jetzt so ist alles okay, aber ich glaube später nein. (männlich, 16 Jahre, Adaptiv-Pragmatische)

( Ich könnte mir vielleicht vorstellen, dass es da Roboter gibt, die einen wecken, die Essen machen, irgendwie sowas. Dass es Chips gibt, die man sich einpflanzen lassen kann. (männlich, 16 Jahre, Materialistische Hedonisten)

( Ich glaube, dass es mit den Handys noch viel krasser wird, dass da noch mehr Sachen dazu kommen. Und dass die größer werden. Wo es dann nur noch Touch-Fläche gibt. Es wird sich natürlich alles weiterbilden. Es kommen immer mehr neue Handys raus weil "Neu ist cool!". Das ist alles nur Geldmacherei. (weiblich, 15 Jahre, Experimentalistische Hedonisten)

\section{Fazit: Digitale Kompetenz ist in den Lebenswelten unterschiedlich ausgeprägt.}

Insgesamt zeigt sich, dass Jugendliche digitale Medien zwar selbstverständlich in ihren Alltag integriert haben und sich auch kaum mehr ein Leben "ohne" vorstellen können; diese Tatsache stimmt sie jedoch nicht unbedingt euphorisch. Sie sehen sich eher als Teil einer Bewegung denn als aktive Treiber der digitalen Entwicklung. Für die 14- bis 17-Jährigen in Deutschland ist der digitale Alltag unhinterfragte Selbstverständlichkeit - digitale Medien sind kein Bonus, sondern Notwendigkeit für die Teilhabe in der Peergroup. "Always on" zu sein ist dabei immer weniger 
ein Status-Symbol. Als Distinktionsmerkmal wird vielmehr die eigene Selbstregulationskompetenz ins Spiel gebracht: an allem Relevanten teilzuhaben, ohne als "internetsüchtig" zu gelten ist die angestrebte soziale Positionierung, insbesondere in den postmodernen Lebenswelten.

Deutlich wird zudem, dass Medienkompetenz keine Frage der Ausstattung ist. Laptopklassen und kostenlosen WLAN-Zugang als hinreichenden Beitrag zur Chancengerechtigkeit zu verstehen, greift deutlich zu kurz. Die Erkenntnisse dieses Kapitels zeigen klar, dass auch bei gleicher Verfügbarkeit von digitalen Medien erhebliche Kompetenz-Unterschiede in den Lebenswelten vorherrschen. Dies hängt auch damit zusammen, dass digitalen Medien lebensweltlich sehr unterschiedliche Bedeutungen und Funktionen zugeschrieben werden - von der reinen Entertainmentzentrale zur Erholung bis hin zur Infrastruktur für Informationsbeschaffung und Berufsplanung.

Open Access Dieses Kapitel wird unter der Creative Commons Namensnennung - Nicht kommerziell 2.5 International Lizenz (http://creativecommons.org/licenses/by-nc/2.5/deed.de) veröffentlicht, welche für nicht kommerzielle Zwecke die Nutzung, Verbreitung und Wiedergabe in jeglichem Medium und Format erlaubt, sofern Sie den/die ursprünglichen Autor(en), den Titel des Werks und die Quelle ordnungsgemäß nennen, einen Link zur Creative Commons Lizenz beifügen und im Falle einer Abwandlung durch einen entsprechenden Hinweis deutlich erkennbar machen, dass Änderungen vorgenommen wurden.

Die in diesem Kapitel enthaltenen Bilder und sonstiges Drittmaterial unterliegen ebenfalls der genannten Creative Commons Lizenz, sofern sich aus der Abbildungslegende nichts anderes ergibt. Sofern das betreffende Material nicht unter der genannten Creative Commons Lizenz steht und die betreffende Handlung nicht nach gesetzlichen Vorschriften erlaubt ist, ist auch für die oben aufgeführten nicht-kommerziellen Weiterverwendungen des Materials die Einwilligung des jeweiligen Rechteinhabers einzuholen. 